OPEN ACCESS

Edited by:

Luca Rastrelli,

University of Salerno, Italy

Reviewed by:

Ina Yosifova Aneva,

Bulgarian Academy of Sciences,

Bulgaria

Wan-Wan Lin,

National Taiwan University, Taiwan

${ }^{*}$ Correspondence:

Yue Shi

shiyue1029@126.com

Specialty section:

This article was submitted to

Ethnopharmacology,

a section of the journal

Frontiers in Pharmacology

Received: 08 June 2021

Accepted: 26 August 2021 Published: 09 September 2021

Citation:

Wu J, Zhang $F$, Ruan $H$, Chang $X$,

Wang J, Li Z, Jin W and Shi Y (2021)

Integrating Network Pharmacology

and RT-qPCR Analysis to Investigate

the Mechanisms Underlying ZeXie

Decoction-Mediated Treatment of

Non-alcoholic Fatty Liver Disease.

Front. Pharmacol. 12:722016.

doi: 10.3389/fphar.2021.722016

\section{Integrating Network Pharmacology and RT-qPCR Analysis to Investigate the Mechanisms Underlying ZeXie Decoction-Mediated Treatment of Non-alcoholic Fatty Liver Disease}

\author{
Jiashuo $W u^{1}$, Fangqing Zhang ${ }^{1}$, Haonan Ruan ${ }^{1}$, Xiaoyan Chang ${ }^{1}$, Jingxun Wang ${ }^{1}$, \\ Zhuangzhuang $\mathrm{Li}^{1}$, Weiyi Jin ${ }^{1,2}$ and Yue Shi ${ }^{1 *}$ \\ ${ }^{1}$ Institute of Medicinal Plant Development, Chinese Academy of Medical Sciences and Peking Union Medical College, Beijing, \\ China, ${ }^{2}$ College of Public Health, Hebei Medical University, Shijiazhuang, China
}

ZeXie Decoction (ZXD) is a traditional Chinese medicine composed of Alisma orientalis (Sam.) Juzep. and Atractylodes macrocephala Koidz. ZXD has been widely used to treat non-alcoholic fatty liver disease (NAFLD). The mechanistic basis for the pharmacological activity of ZXD, however, remains poorly understood. In this study, we used a network pharmacology approach and investigated the association between ZXD and NAFLD. We identified the active ingredients of ZXD and screened the potential targets of these ingredients, after which a database of relevant NAFLD-related targets were constructed and several enrichment analyses were performed. Furthermore, the ethanol and aqueous extracts of ZXD were prepared and experimental pharmacology validation was conducted using RT-qPCR of the non-alcoholic fatty liver disease (NAFLD) model in Sprague-Dawley (SD) rats. As a result, a herb-compound-target-pathway network model was developed, and HMGCR, SREBP-2, MAPK1, and NF- $\kappa$ Bp65 targets were validated. The gene expression results of these four targets were consistent with those of the network pharmacology prediction. Using an integration strategy, we revealed that ZXD could treat NAFLD by targeting HMGCR, SREBP-2, MAPK1, and NF- $\kappa$ Bp65.

Keywords: ZeXie decoction, network pharmacology, RT-qPCR analysis, non-alcoholic fatty liver disease, integrating strategy

\section{INTRODUCTION}

Rising obesity rate has partially been linked to the increasing prevalence of non-alcoholic fatty liver disease (NAFLD), characterized by hepatic steatosis unrelated to alcohol consumption (Than and Newsome, 2015). More than $25 \%$ of people suffer from NAFLD worldwide. A significant cause of human morbidity, NAFLD, can progress to severe conditions, including fibrosis, cirrhosis, and hepatoma (Tandon, 2013; Angulo et al., 2015). Treatment options for NAFLD are limited and are centered mainly around bariatric surgery and lifestyle changes.

Traditional Chinese medicine formulations (TCMFs) are traditional medicines that are used clinically for many years. ZeXie Decoction (ZXD) is one of the TCMFs composed of Alismatis Rhizoma (ZX, the rhizome of Alisma orientale (Sam.) Juzep.) and Atractylodis Macrocephalae 


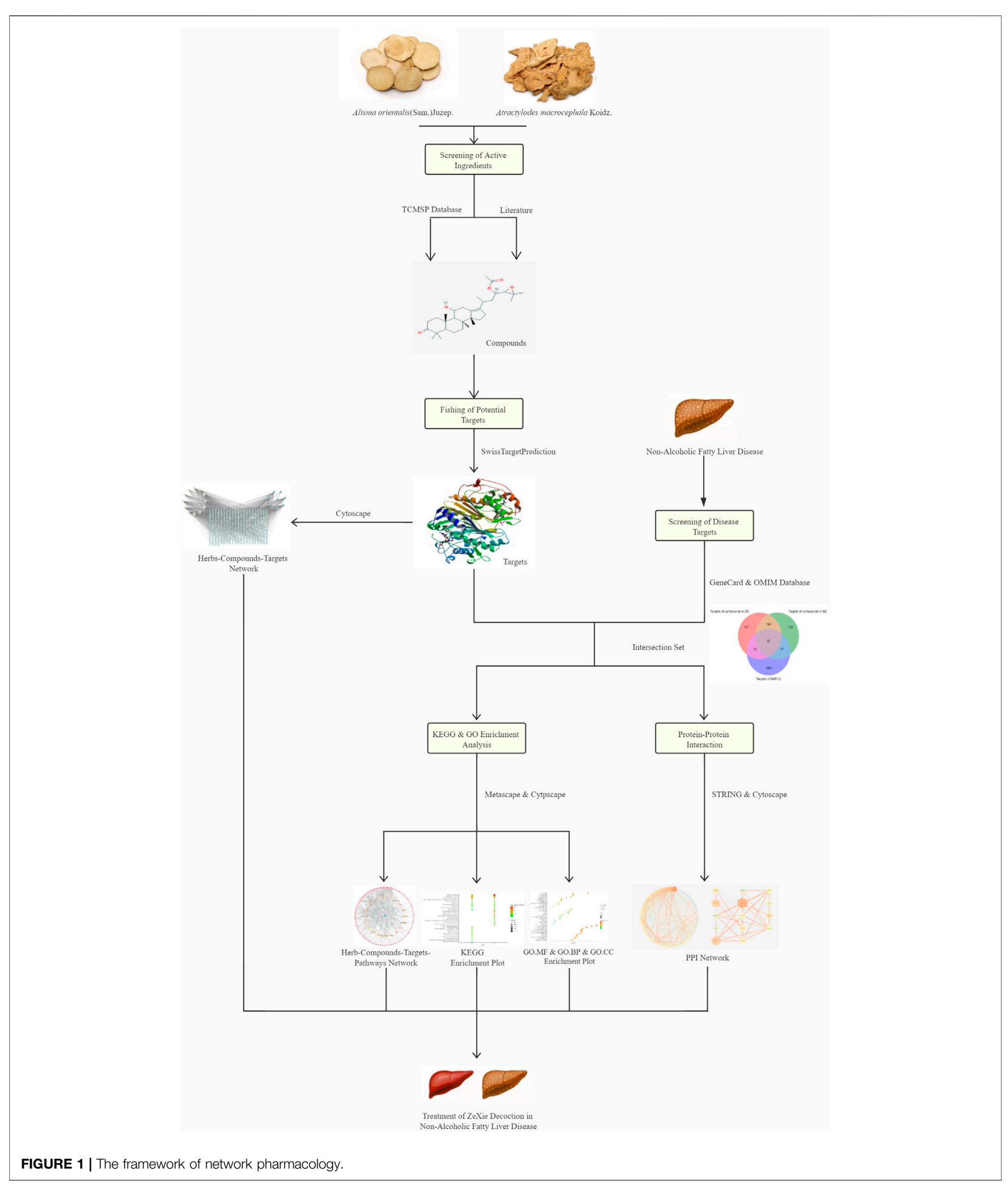

Rhizoma (BZ, the rhizome of Atractylodes macrocephala Koidz.) in the ratio of 5:2 on a dry weight basis. ZX contains bioactive triterpenoid, including alisol A, alisol B, alisol A 23-acetate, alisol
A 24-acetate, alisol B 23-acetate and so forth. BZ contains bioactive triterpenoid, including atractylenolide I, atractylenolide II, atractylenolide III and so forth 
TABLE 1 | PCR primer design.

\section{Gene name}

HMGCR

SREBP-2

MAPK

$\mathrm{NF}-\kappa \mathrm{B}$
Forward primer $\left(5^{\prime}-3^{\prime}\right)$

TTGCACGTCTACAGAAACTTCATAC

TGTGACCTGCTACTGTCGCTA

ATTACGACCCGAGTGACGAG

CGACGCATTGCTGTGCCTTC
Reverse primer $\left(5^{\prime}-3^{\prime}\right)$

CCTGACCTGGACTGGAAACG

AACACCTTGCGGTATGCTG

CAAAGTGGATAAGCCAAGAC

ATGGTGCTCAGGGATGACGTAAAG

TABLE 2 | Information of ingredients in ZXD.

\begin{tabular}{|c|c|c|c|c|}
\hline Id & CID & Chemical name & Source & Literature \\
\hline Z1 & 14036813 & Alisol C Monoacetate & $Z X$ & Li et al. (2016); Chang et al. (2021) \\
\hline Z2 & 101306923 & Alisol C & $Z X$ & Li et al. (2016); Chang et al. (2021) \\
\hline Z3 & 14036811 & Alisol B 23-Acetate & $Z X$ & Szklarczyk et al. (2017); Chang et al. (2021) \\
\hline Z4 & 15558620 & Alisol B & $Z X$ & Alex (2019); Chang et al. (2021) \\
\hline Z5 & 222284 & Sitosterol & $Z X$ & Chang et al. (2021) \\
\hline Z6 & 5283469 & 1-Monolinolein & $Z X$ & Chang et al. (2021) \\
\hline $\mathrm{Z7}$ & 15558616 & Alisol A & $Z X$ & Gong et al. (2018) \\
\hline Z8 & 74344393 & Alisol A 24-Acetate & $Z X$ & Szklarczyk et al. (2017); Wu et al. (2018) \\
\hline Z9 & 70690607 & Alisol A 23-Acetate & $Z X$ & Hong et al. (2010) \\
\hline Z10 & 102004739 & 25-Anhydroalisol F & $Z X$ & Bi et al. (2017) \\
\hline Z11 & 76310822 & Alisol F & $Z X$ & Bi et al. (2017) \\
\hline Z12 & 70688546 & Alisol M 23-Acetate & $Z X$ & Yang et al. (2019) \\
\hline B1 & 5321018 & Atractylenolide I & $\mathrm{BZ}$ & Hong et al. (2010); Tang et al. (2017) \\
\hline B2 & 14448070 & Atractylenolide ॥ & $\mathrm{BZ}$ & Hong et al. (2010) \\
\hline B3 & 155948 & Atractylenolide III & $\mathrm{BZ}$ & Xu et al. (2016a) \\
\hline B4 & 132941086 & 14-Acetyl-12-Senecioyl-2E,8Z,10E-Atractylentriol & $\mathrm{BZ}$ & Chang et al. (2021) \\
\hline B5 & 73170 & $\alpha$-Amyrin & $\mathrm{BZ}$ & Chang et al. (2021) \\
\hline B6 & 15976101 & (24S)-24-Propylcholesta-5-Ene-3 $\beta$-Ol & $\mathrm{BZ}$ & Chang et al. (2021) \\
\hline B7 & 14448075 & 8ß-Ethoxy Atractylenolide III & $\mathrm{BZ}$ & Chang et al. (2021) \\
\hline
\end{tabular}

The CID of compound was obtained from PubChem database.

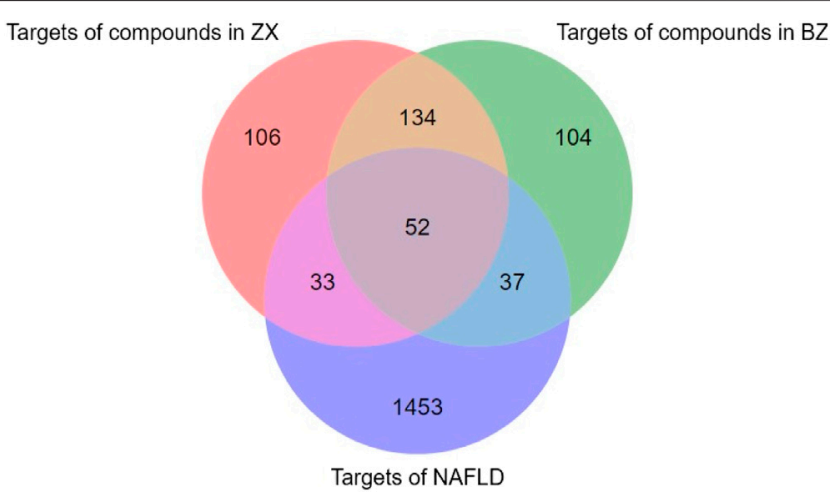

FIGURE 2 | The venn diagram of targets in ZX, BZ and NAFLD. Totally 1575 NAFLD-related targets were identified, of which 122 targets overlapped with our list of ZXD targets.

(Hoang et al., 2016; Liu et al., 2019). Several mechanistic studies have found that ZXD, ZX, BZ, and other active derivatives could treat NAFLD through the modulation of insulin, and PI3K/AKT/ NF- $\kappa \mathrm{B}$ and PPAR pathways, thereby suppressing inflammation and lowering lipid levels in treated individuals (Dan et al., 2011; Song et al., 2014; Li et al., 2016; Bi et al., 2017; Tang et al., 2017; Wu et al., 2018). ZXD is a promising complementary therapy for the treatment of NAFLD.
TCMFs are typically designed as multi-component multitarget pharmaceutical agents, in contrast to modern drugs typically designed to target a single protein (Zhang et al., 2019). Presently, the molecular mechanisms underlying the efficacy of many TCMFs remain uncertain. In the past, experimental model systems were leveraged for pharmacological studies of TCMFs. However, developments in systems biology have led to more widespread use of network pharmacology-based analyses of traditional medicine preparations (Lee et al., 2018; Zuo et al., 2018; Huang et al., 2020). Network pharmacology tools offer an ideal opportunity to understand the complex mechanisms through which TCMFs function in vivo.

In our previous work (Chang et al., 2021), we studied ethanol and aqueous extracts of ZXD using ultraperformance liquid chromatography coupled with mass spectrometry to identify the major components of ZXD. Based on this work, published literature, and database sources, we developed a herb-compound-target-pathway network model and performed enrichment analysis to investigate the relationship between $\mathrm{ZXD}$ and NAFLD treatment. The network pharmacology framework is depicted in Figure 1. Basing on the non-alcoholic fatty liver disease (NAFLD) model in Sprague-Dawley (SD) rats, the prediction of the mechanism was validated by RT-qPCR analysis in vivo. 


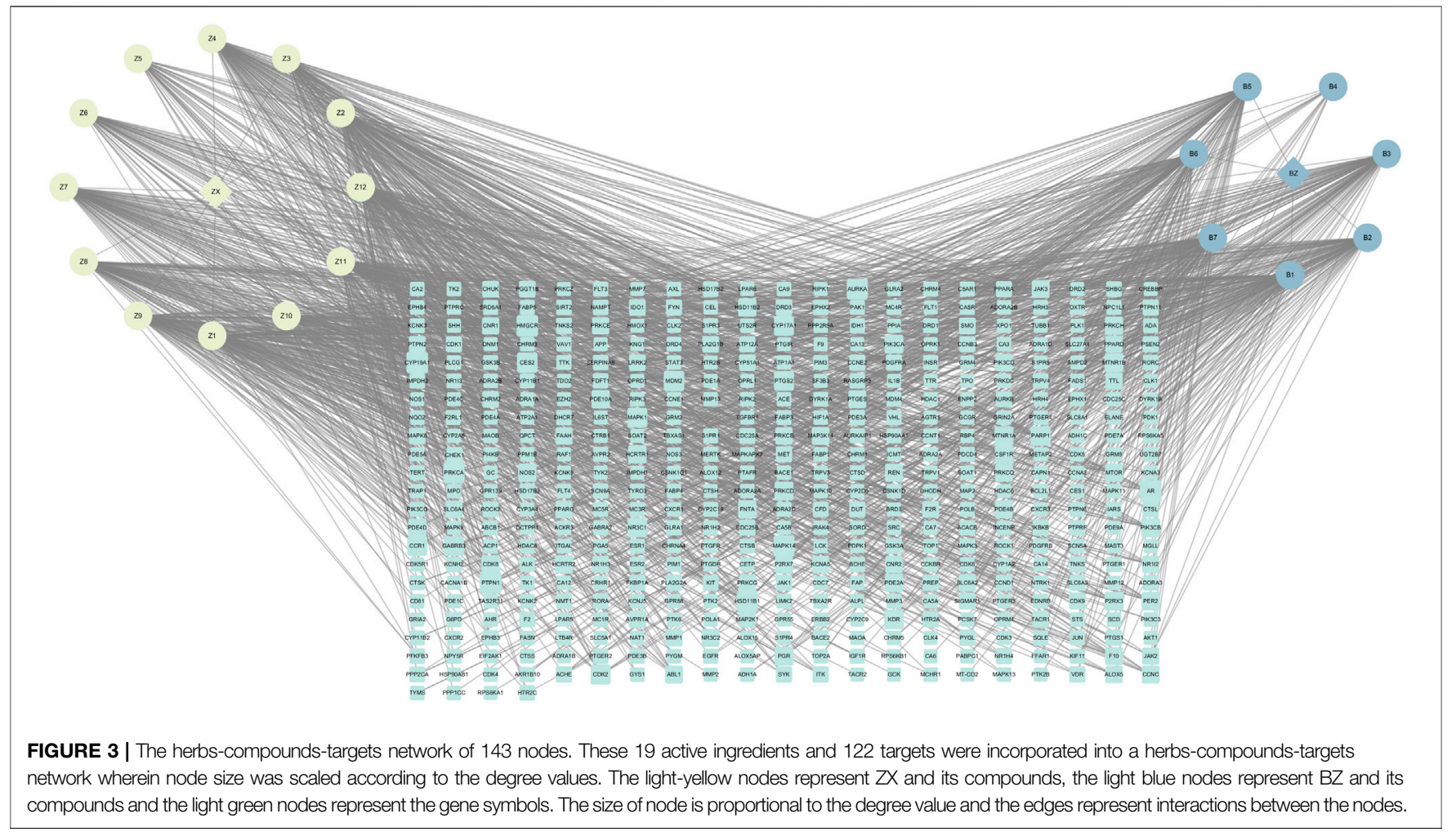

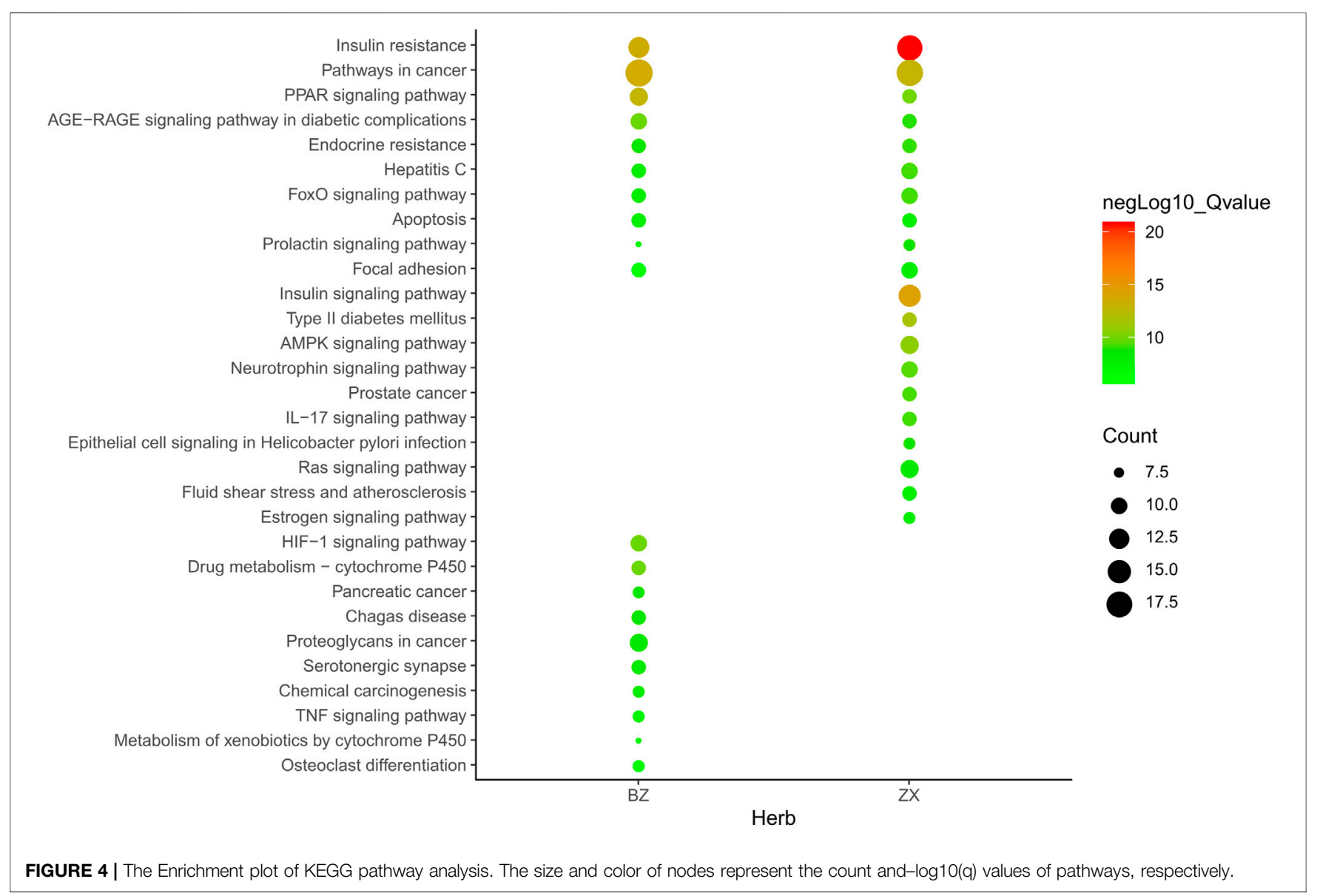




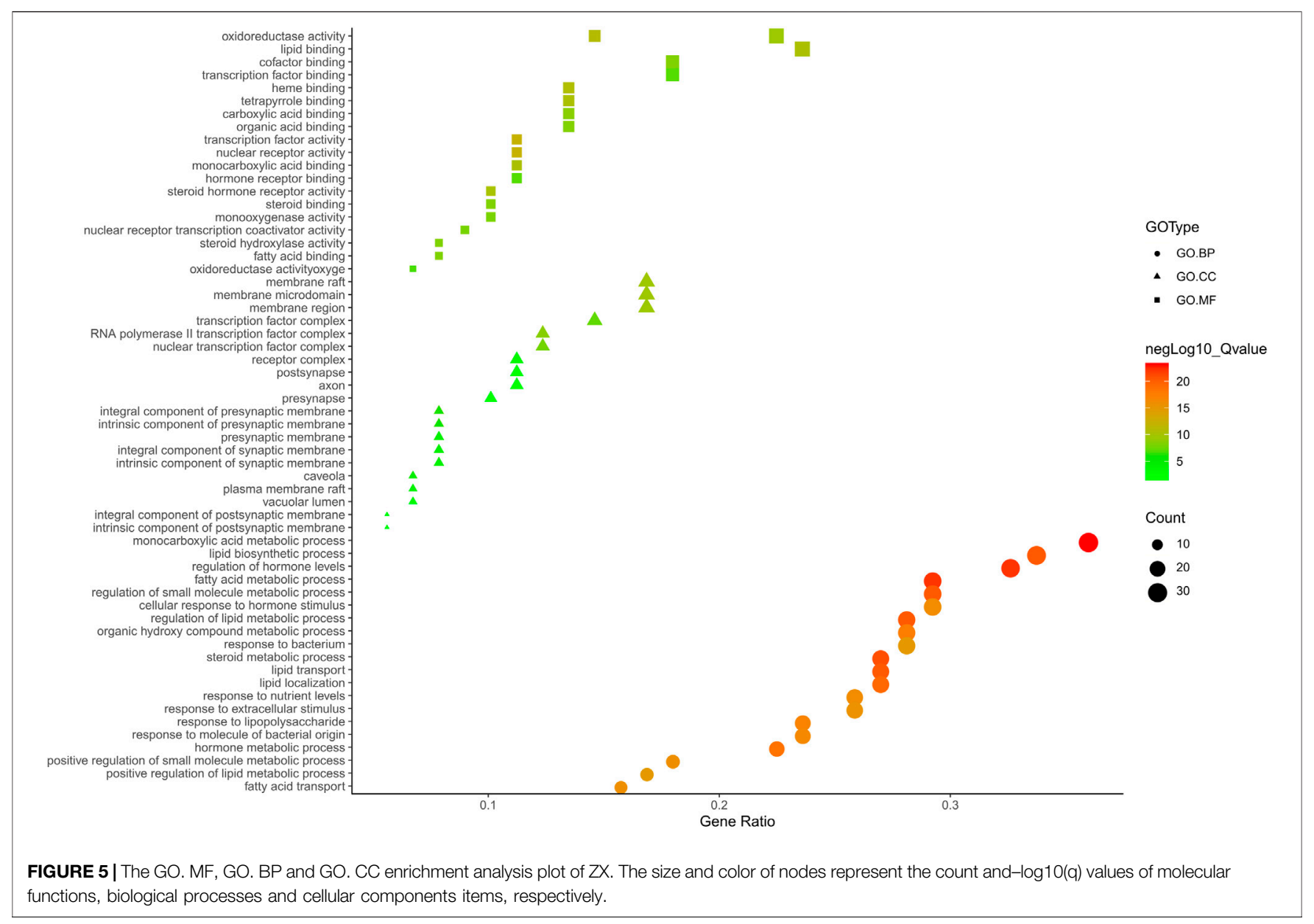

\section{MATERIALS AND METHODS}

\section{Experimental Animals}

A total of 72 male SD rats weighting $200 \pm 20$ g (Beijing Vital River Laboratory Animal Technology Co. Ltd.) were housed in a room at a constant temperature of $23 \pm 3^{\circ} \mathrm{C}$, a constant humidity of $50 \pm 10 \%$, under a $12 \mathrm{~h}$ light/dark cycle. The rats were fed UVdisinfected fodder and drinking water. The animal experiment was approved by the Laboratory Animal Center, Institute of Medicinal Plant Development, Chinese Academy of Medical Sciences and Peking Union Medical College. The rats were adaptively bred for 1 week in the facility before the experiments.

\section{Reagents}

The dried rhizomes of $Z X$ (Batch number: DD6081) and $B Z$ (Batch number: DD8061) were purchased from Beijing Huamiao Pharmaceutical Co., Ltd. (Beijing, China). Their qualities were in accordance with the Chinese Pharmacopoeia. The specimens of ZX and BZ have been preserved in the Institute of Medicinal Plant Development, Chinese Academy of Medical Sciences and Peking Union Medical College.

PrimeScript RT reagent kit, TRIzol reagent, and DNA marker were purchased from TaKaRa Bio (Beijing, China). The total cholesterol (TC), triglycerides (TG), aspartate aminotransferase (AST), alanine aminotransferase (ALT) assay kit were purchased from Beijing Solarbio Science and Technology Co. Ltd (Beijing, China). Urethane, pioglitazone hydrochloride $(\mathrm{PH})$ and paraformaldehyde were purchased from Macklin Reagent Company (Shanghai, China).

\section{Sample Preparation}

The samples of ZXD were prepared by combining $\mathrm{ZX}$ and $\mathrm{BZ}$ in a $5: 2$ ratio $(w / w)$. The samples were crushed and refluxed thrice with 10 -times the volume of $95 \%$ ethanol for $2 \mathrm{~h}$. The filtrates were then pooled and dried using a rotary evaporator under vacuum, yielding $14.29 \%$ ethanol extract.

\section{Qualitative Analysis}

The comparative study of the ethanol and aqueous extracts of ZXD using UPLC-DAD-Q-TOF-MS was performed in our previous study to simultaneously identify the major chemical components in these two extracts (Chang et al., 2021). This analysis was conducted using an Waters ACQUITY UPLC system with a binary solvent delivery system and an autosampler. An Waters Acquity UPLC HSS T3 column $(1.7 \mu \mathrm{m}, 2.1 \mathrm{~mm} \times 100 \mathrm{~mm}$; Waters, MA, United States $)$ was 


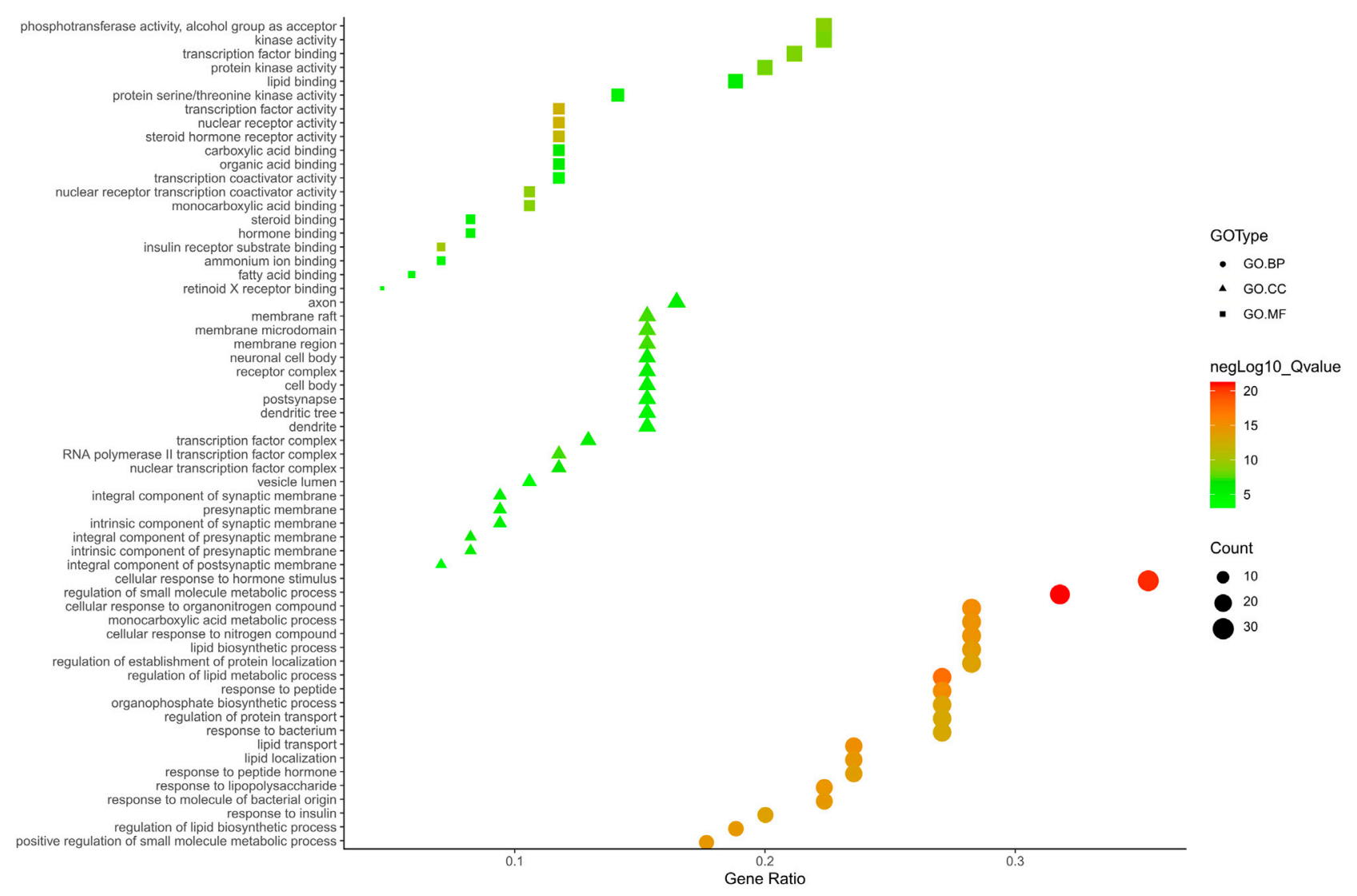

FIGURE 6 | The GO. MF, GO. BP and GO. CC enrichment analysis plot of BZ. The size and color of nodes represent the count and-log10(q) values of molecular functions, biological processes and cellular components items, respectively.

used to conduct this analysis at $30^{\circ} \mathrm{C}$. Acetonitrile (A) and formic acid in water $(0.1 \%, v / v)$ (B) were used as the mobile phase in this analysis, with $0.2 \mathrm{ml} / \mathrm{min}$ being selected as the flow rate. A linear gradient elution approach was used with the following settings: 0-10 min, 0-1\% A; $10-15 \mathrm{~min}, 1-3 \%$ A; $15-20 \mathrm{~min}, 3-10 \% \mathrm{~A}$; 20-25 min, $10-15 \%$ A; $25-30 \mathrm{~min}, 15-20 \%$ A; $30-35 \mathrm{~min}$, $20-30 \%$ A; $35-40 \mathrm{~min}, 30-60 \%$ A; $40-45 \mathrm{~min}, 60-80 \%$ A; 45-50 min, $80-90 \%$ A; $50-55 \mathrm{~min}, \quad 90-100 \%$ A. A $2 \mu \mathrm{L}$ injection volume was used for all analysis, with $15^{\circ} \mathrm{C}$ as the injection temperature.

A Q-TOF analyzer in the SYNAPT G2 HDMS system (Waters, Manchester, United Kingdom) was used for tandem MS analyses. Positive ionization mode was used to acquire ESI spectra in the $\mathrm{m} / \mathrm{z} 50-1,200$ mass range with the following settings: a $120^{\circ} \mathrm{C}$ source temperature; $3.0 \mathrm{kV}$ as the chosen capillary voltage; a desolvation gas temperature of $450^{\circ} \mathrm{C}$ and a gas flow rate of $800 \mathrm{~L} /$ h; a $40.0 \mathrm{~V}$ sampling cone voltage and a $50 \mathrm{~L} / \mathrm{h}$ cone gas rate; $10-40 \mathrm{eV}$ as the collision energy. The Masslynx 4.1 software package was used to acquire all data.

\section{The Study of Network Pharmacology Bioactive Ingredient Identification}

The widely used Traditional Chinese Medicine System Pharmacology database and Analysis Platform (Ru et al., 2014)
(TCMSP, https://tcmspw.com/tcmsp.php) was utilized to screen the active ingredients within ZX, BZ, and ZXD. In total, 101 herbal ingredients were identified using the keywords "Alisma Orientale (Sam.) Juzep." and "Atractylodes Macrocephala Koidz.". The potential active ingredients were then selected using an oral bioavailability (OB) threshold of $\geq 30 \%$ and a drug-likeness (DL) threshold of $\geq 0.18$ (Leeson and SPRINGTHORPE, 2007; Xu et al., 2012). In addition, any compounds in ZXD, ZX, or BZ that have previously been reported to be highly pharmacologically active were also included in this analysis (Wu et al., 2021). Two-dimensional (2D) structures of all compounds were obtained from the PubChem database (https://pubchem.ncbi.nlm.nih.gov/) and used for target prediction efforts.

\section{Target Prediction}

TheSwissTargetPrediction platform (David et al., 2013) (http://www.swisstargetprediction.ch/index.php) was utilized to identify the putative targets of the compounds retrieved from the PubChem database. The Swiss library contains over 3,000 proteins and 370,000 active ingredients, making it ideal for such efforts. Files of the identified bioactive ingredients with the suffix "SDF" were used as inputs for the SwissTargetPrediction platform, and probable macromolecular targets of these compounds were 


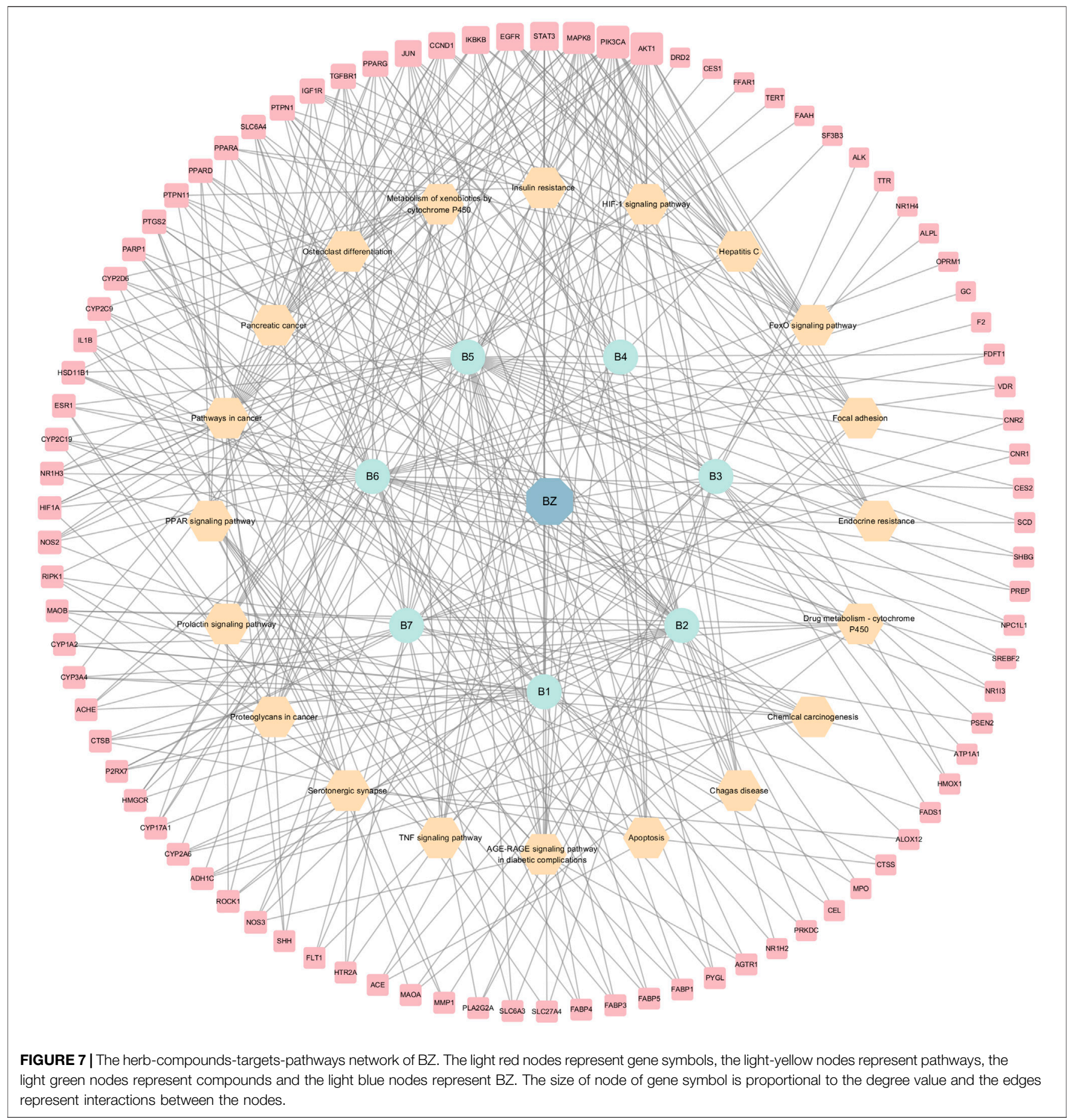

identified based on structural similarity. Only the SwissTargetPrediction platform was used for this predictive analysis to ensure the clarity of results. The species was set to Homo sapiens for this analysis and all subsequent analyses.

\section{NAFLD-Related Target Identification}

Annotated and predicted NAFLD-related targets were identified using the Online Mendelian Inheritance in Man
(OMIM, https://omim.org/) (Hamosh et al., 2002) and GeneCards (https://www.genecards.org/) (Stelzer et al., 2016) databases. We used "Non-alcoholic fatty liver" and "Non-alcoholic fatty liver disease" as search terms and integrated the search results to develop a NAFLD-specific disease target database. NAFLD-related targets that were also identified as putative targets of ZXD bioactive ingredients were retained for further analysis. 


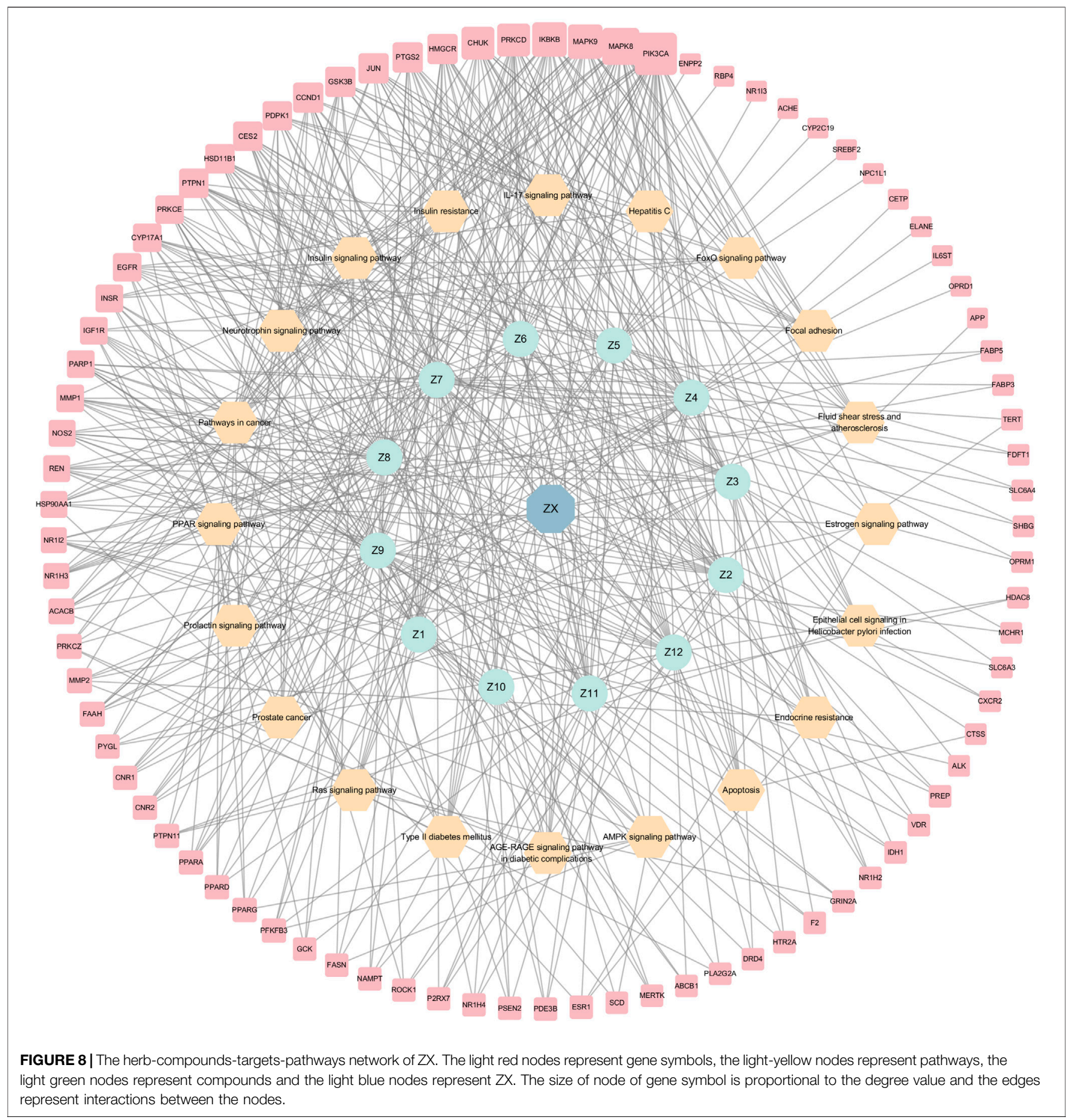

\section{Network Construction and Enrichment Analyses}

Cytoscape 3.7.2 (Shannon et al., 2003) (https://cytoscape.org/) was used to construct a herb-compound-target network, with nodes corresponding to herbs, compounds, and targets and edges representing between-node interactions. Node sizes were scaled based on degree values determined using Cytoscape Network Analyzer such that smaller nodes were associated with smaller degree values.
The potential functional roles of targets overlapping between ZXD and NAFLD target lists were evaluated via Metascape (Zhou et al., 2019) (https://metascape.org/), Kyoto Encyclopedia of Genes and Genomes (KEGG) pathway analyses, and Gene Ontology (GO) enrichment analyses. All genes were used as the enrichment background for KEGG analyses, with significantly enriched terms meeting the following criteria: count $\geq 3, p<0.01$, enrichment factor $>1.5$. These terms were 


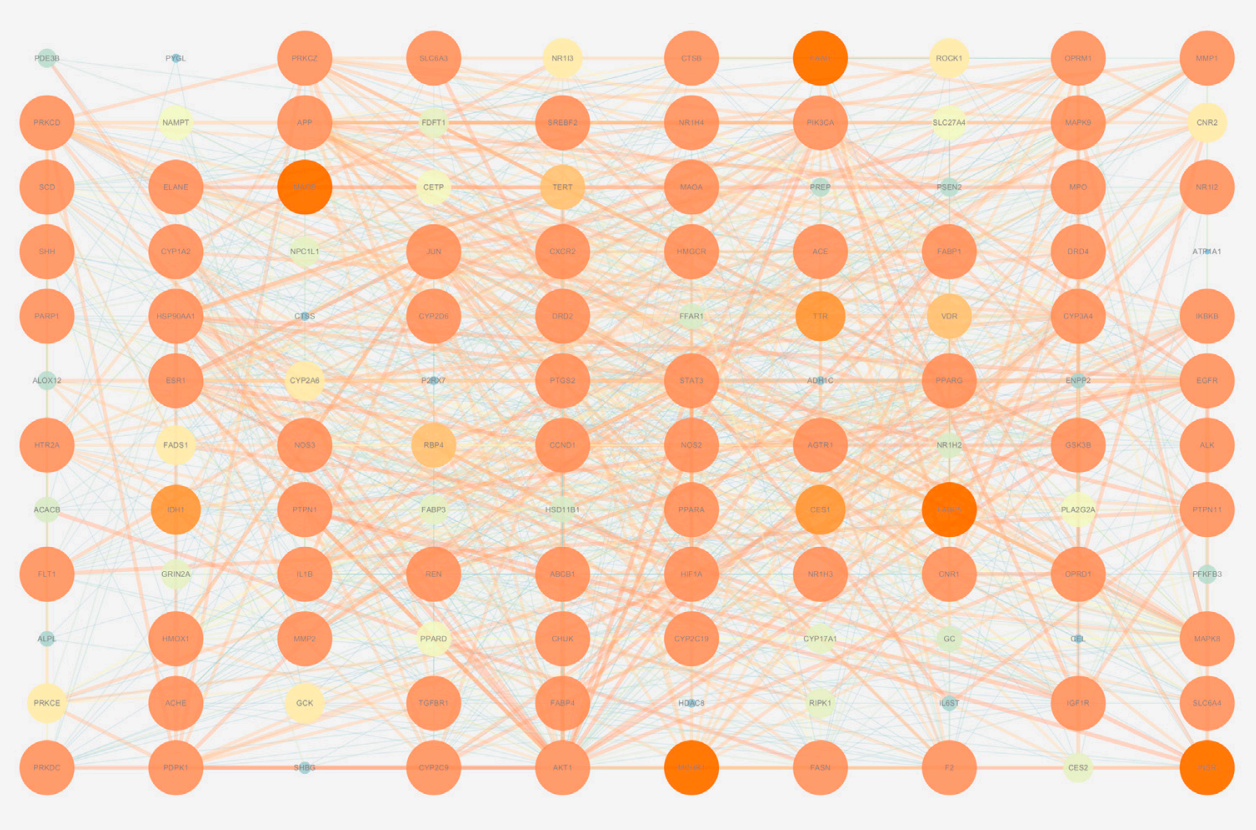

FIGURE 9 | The PPI network of all matched targets. The size of nodes represents their degree values and the width of edges represents interactions between the nodes. The color represents their degree values and combined scores, which changes from blue to orange represent values from low to high.

grouped and clustered based on the membership similarity. For GO analyses, the molecular functions (MFs), biological processes (BPs), and cellular components (CCs) for which these targets were enriched were identified and visualized using enrichment plots.

The ZX and BZ herb-compound-target-pathway networks were then plotted based on the results of the above enrichment analyses.

\section{Protein-Protein Interaction Analysis}

Protein-protein interaction (PPI) analysis was conducted to identify which ZXD targets are most crucial in the context of disease pathology. STRING 11.0 (Szklarczyk et al., 2017) (https:// string-db.org/) database was used to conduct PPI analysis of overlapping ZXD and NAFLD targets and KEGG pathway analysis. For the PPI analysis, active interaction sources were text mining, experiments, databases, co-expression, neighborhood, gene fusion, and co-occurrence. The analysis range was only set to input proteins, and output obtained in the form of files with the suffix "TSV" was visualized using Cytoscape 3.7.2; NetworkAnalyzer was used to assess the degree and combined scores.

All macromolecular targets were retrieved from the Uniprot database (Alex, 2019) (https://www.uniprot.org/) to confirm their gene symbols. The plots described above were designed based on these symbols to ensure statistical normalization.

\section{Experimental Pharmacology Validation Experimental Design}

Seventy-four male SD rats were randomly divided into two groups according to their diets, including blank $(n=13)$ and model group $(n=61)$. The blank group rats were fed with normal chew diets (NCD, Beijing Hfk Bioscience Co., Ltd.), and the model group rats were fed with high-fat diets containing $41 \%$ fat (HFD, Beijing Hfk Bioscience Co., Ltd.) for 12 weeks to induce NAFLD. After 12 weeks of modeling, one rat liver tissue from each group was used for histopathological evaluation to validate the NAFLD model. Afterwards, a 4-weeks intervention period with ZXD and positive control drug started. Rats from model group were randomly and equally assigned to five subgroups: vehicle, positive control, low-dose, middle-dose and high-dose groups ( $\mathrm{n}=12$ each). The vehicle group and positive control group rats were orally administered distilled water and $10 \mathrm{mg} / \mathrm{kg} / \mathrm{d} \mathrm{PH}$, respectively. The low-dose, middle-dose and high-dose group rats were orally administered 100, 200 and $400 \mathrm{mg} / \mathrm{kg} / \mathrm{d}$ ZXD, respectively. The doses of ZXD and PH were selected based on the previous studies (Hong et al., 2010; Gong et al., 2018; Yang et al., 2019). During the 4 -week intervention period, the blank group rats were fed with NCD and the remaining five group rats were fed with HFD. Animals were given free access to feed and drink. The animal weights and food consumption were monitored daily. At the end of 


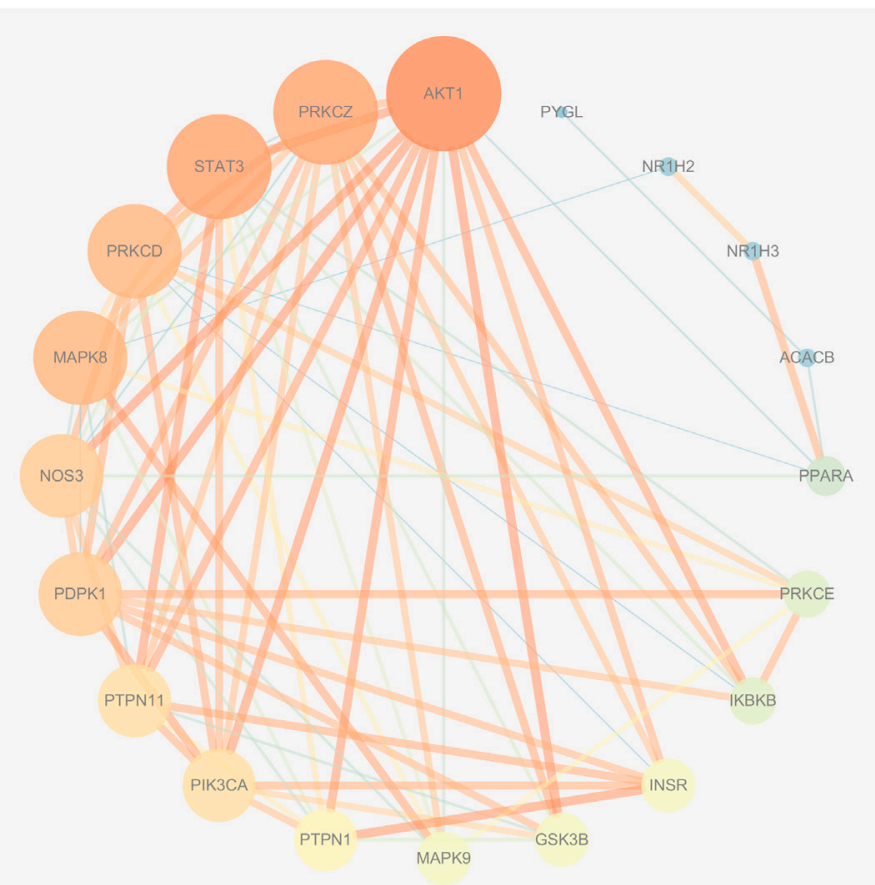

FIGURE 10 | The PPI network of insulin-related targets. The size of nodes represents their degree values and the width of edges represents interactions between the nodes. The color represents their degree values and combined scores, which changes from blue to orange represent values from low to high.

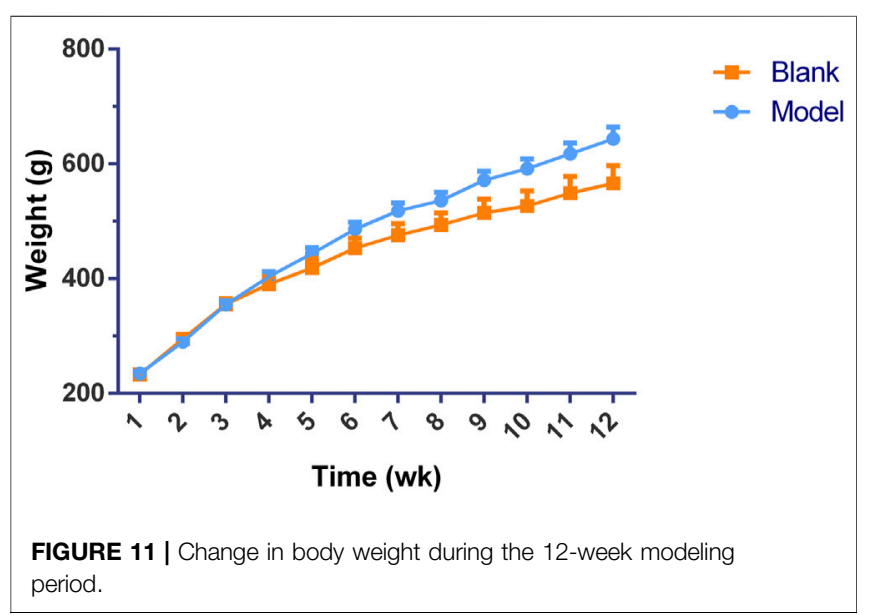

intervention period, animals were fasted for $16 \mathrm{~h}$ and then anesthetized with an intraperitoneal injection of urethane $(20 \%, 1 \mathrm{~g} / \mathrm{kg})$. The biological samples (blood, liver and urine) were collected and stored at $-80^{\circ} \mathrm{C}$ to conduct the histopathological evaluation and serum biochemical assay.

\section{Histopathological Evaluation}

\section{Hematoxylin and Eosin (HE) Staining}

Fresh liver tissues were fixed with $4 \%$ paraformaldehyde for $24 \mathrm{~h}$. The fixed tissues were then embedded in paraffin, cut into thin slices and stained with HE. Light microscopy was used to determine the histopathological changes.

\section{Oil Red O (ORO) Staining}

Frozen liver tissues were cut into thin sections and then mounted on glass slides. The slides were fixed in pre-cold $4 \%$ paraformaldehyde and rinsed with distilled water. The rinsed slides were placed in isopropanol solution and then incubated in ORO solution for $10 \mathrm{~min}$. Afterwards, the ORO solution was removed and the slides were rinsed with running tap water for $5 \mathrm{~min}$. Light microscopy was used to determine the histopathological changes.

\section{Serum Biochemical Assays}

The blood samples were collected and left standing for $30 \mathrm{~min}$. The samples were centrifuged for $10 \mathrm{~min}$ at 3,000 g to separate the serum for biochemical assays. The total cholesterol (TC), triglycerides (TG), aspartate aminotransferase (AST), alanine aminotransferase (ALT) levels were measured according to the kit instructions.

\section{RT-qPCR}

RT-qPCR was then performed to measure the hepatic expression of the predicted NAFLD-related genes. Total RNA was collected with the RNA isolation kit according to the instructions. The purity of the collected RNA was detected by the NanoDrop ND-2000 spectrophotometer, 

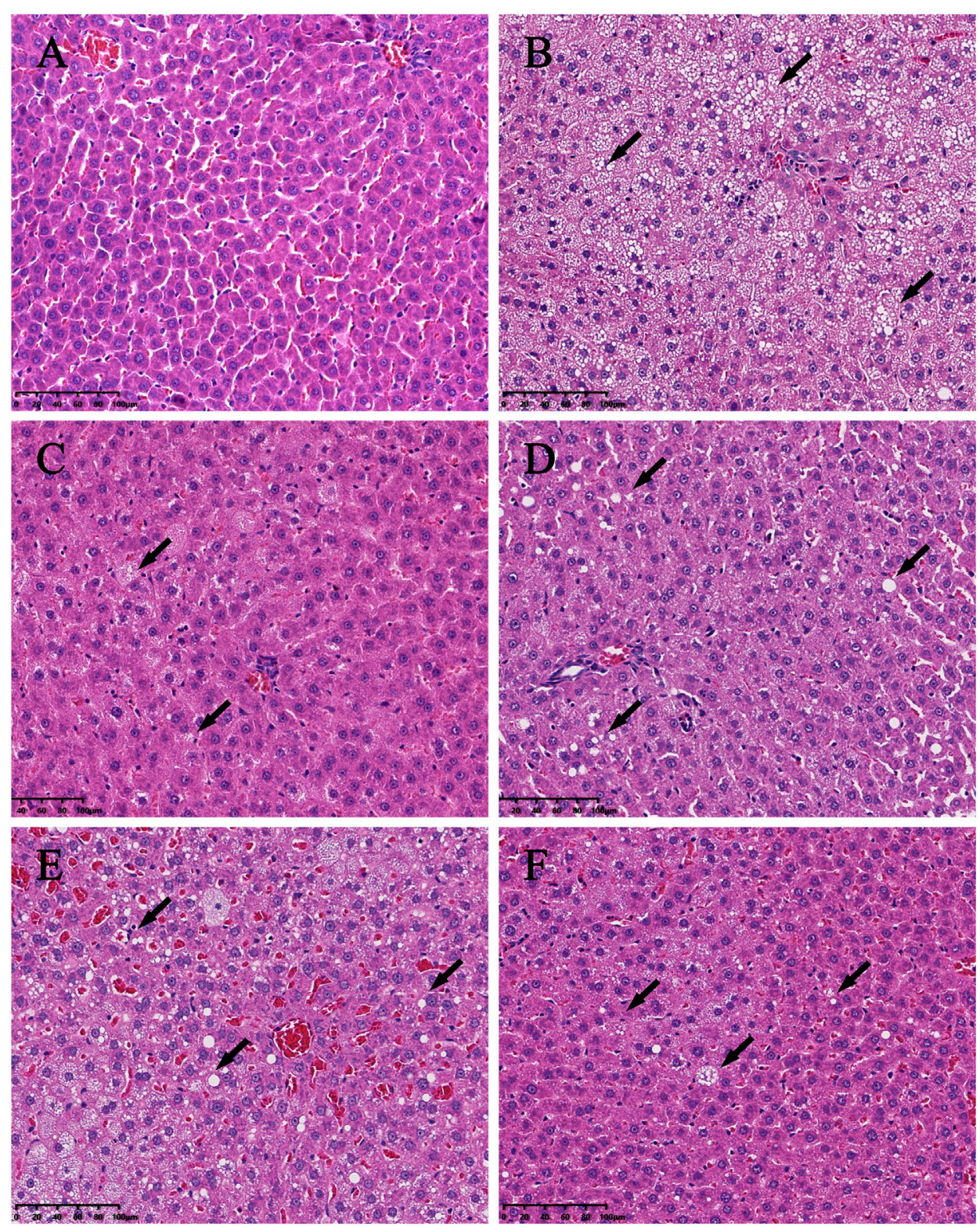

FIGURE 12 | Histopathologic evaluation of liver tissues by HE staining (200x). (A) blank; (B) model; (C) positive control; (D) high-dose; (E) middle-dose; (F) lowdose. The lipid drops are indicated by arrows.

and the 260/280 ratios were calculated. PrimeScript ${ }^{\mathrm{TM}} \mathrm{RT}$ reagent kit with gDNA eraser was used to carry out the reverse transcription reactions. The Realtime-PCR was performed on the ABI7500 fast system. The calculation of relative gene expression was based on the $2^{-\Delta \Delta \mathrm{CT}}$ method. The primers used in the experiments are listed in Table $\mathbf{1}$.

\section{Statistical Analysis}

Statistical analysis was performed using SPSS 23.0 software, and the results are presented as mean $\pm \mathrm{SD}$. One-way analysis of variance was used for comparison, and $p$-value $<0.05$ was considered statistically significant. The data graphs were generated using GraphPad Prism 8.

\section{RESULTS}

\section{Active Ingredients of ZXD}

In our initial search of the TCMSP database, we identified 101 active ingredients of potential relevance. Of these, we selected six ingredients in $\mathrm{ZX}$ (Z1 to Z6) and four ingredients in BZ (B4 to B7) based upon their OB and DL values. In addition, we selected 


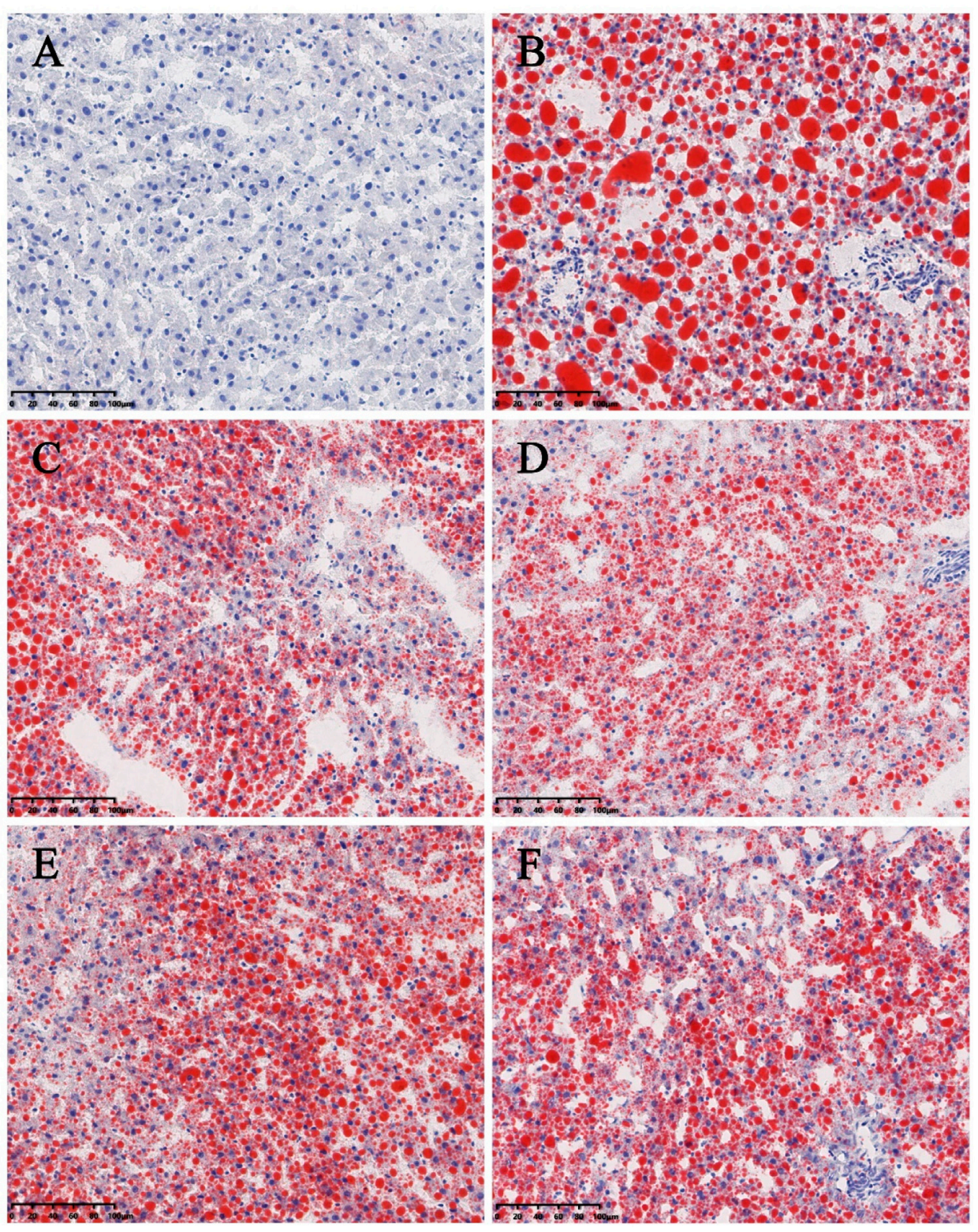

FIGURE 13| Histopathologic evaluation of liver tissues by ORO staining (200x). (A) blank; (B) model; (C) positive control; (D) high-dose; (E) middle-dose; (F) lowdose. Compared with the model group rats, the treated group rats reduced accumulation of lipid droplet remarkably.

nine ingredients (Z7 to Z12 and B1 to B3) that have previously been reported to exhibit a high degree of pharmacological activity in ZXD preparations (Lin, 2012; Xu et al., 2016a; Chao et al., 2016; Song et al., 2017; Xu et al., 2018; Ho et al., 2019; Wu et al., 2021). These ingredients were numbered Z1-Z12 and B1-B7, and compound CID was confirmed with the Pubchem database (Table 2).

\section{Identification of ZXD and NAFLD Targets}

These 19 active compounds were next introduced into the Swiss platform to identify putative macromolecular targets. We identified $325 \mathrm{ZX}$ targets and $327 \mathrm{BZ}$ targets, and 186 overlapping targets (Supplementary Table 1). In addition, the
OMIM and GeneCards databases were used to identify 1575 NAFLD-related targets, of which 122 targets overlapped with our list of ZXD targets (Supplementary Table 2) (Figure 2). These overlapping targets were retained, and corresponding Uniprot IDs were determined and used to identify corresponding gene symbols for each target.

These 19 active ingredients and 122 targets were incorporated into a herbs-compounds-targets network wherein node size was scaled according to the degree values (Figure 3). The targets with the highest degree values included HMGCR, MAPK1, and SREBP-2, suggesting that these targets may play critical roles in the context of NAFLD treatment. 

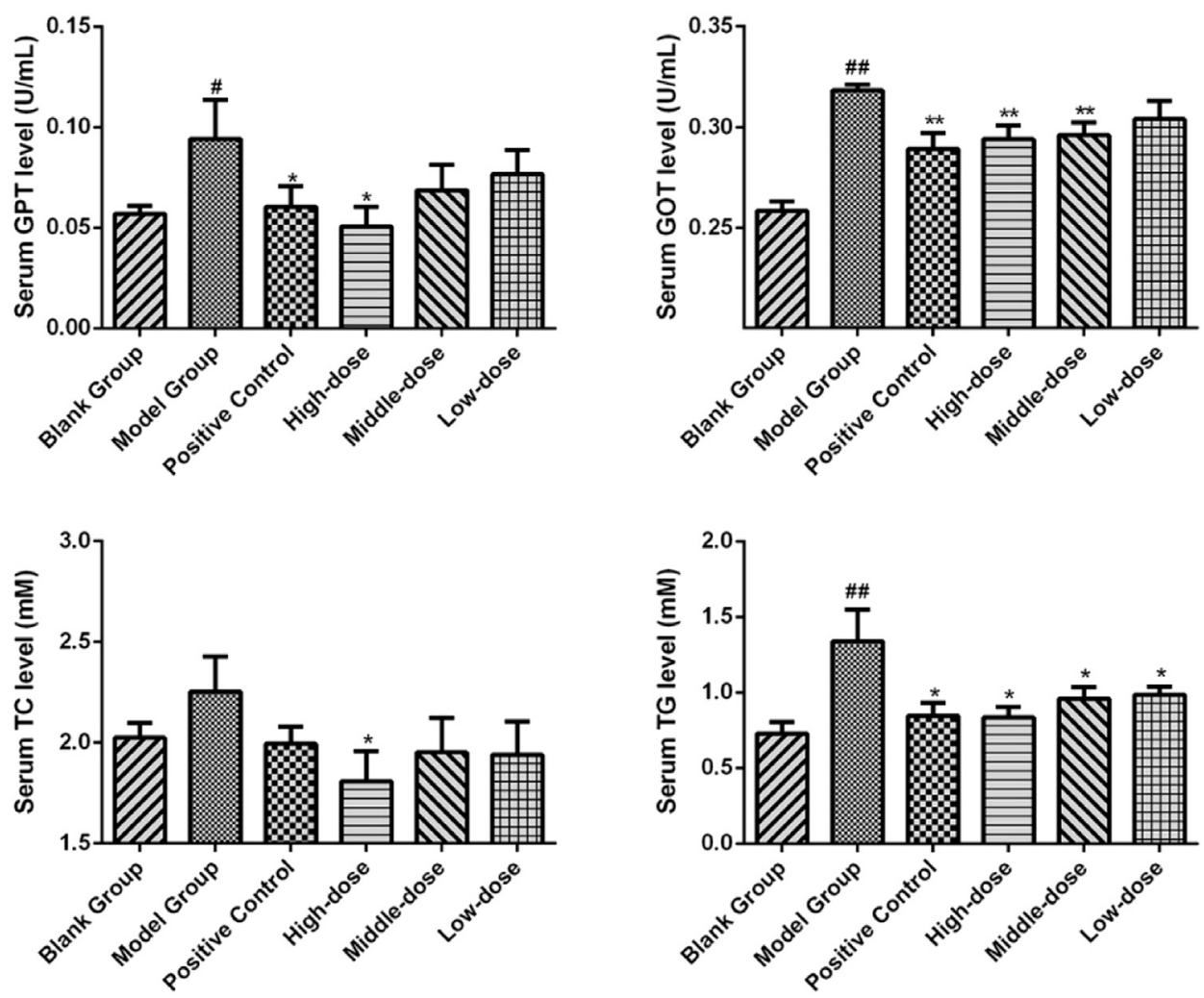

FIGURE 14 | The serum biochemical parameter of rats after the 4-weeks intervention period. ${ }^{*} p<0.05$ and ${ }^{* \star} p<0.01$ vs. model group rats; \#p $<0.05$ and \#\#p $<$ 0.01 vs. blank group rats.

\section{Functional Enrichment and PPI Network Analyses}

Metascape platform was used to carry out KEGG pathway and GO enrichment analyses of the 122 overlapping ZXD and NAFLD targets. Results were ranked based on ascending q-values, corresponding to the degree of correlation with these targets, and the top 20 of these enriched pathways were plotted (Supplementary Table 3) (Figures 4-6). These targets were enriched for the lipid metabolism GO BP, suggesting that this pathway may be linked to the pharmacological activity of ZXD. Other identified targets associated with this pathway were also retained for further analysis (Supplementary Table 4).

KEGG analyses revealed that insulin-related signaling and $\mathrm{PI} 3 \mathrm{~K} / \mathrm{AKT} / \mathrm{NF}-\kappa \mathrm{B}$ pathways are closely associated with ZXDmediated NAFLD treatment. Using these results, we constructed a herb-compound-target-pathway network (Figures 7, 8).

To explore the interactions among the above targets in-depth, we constructed a PPI network using the STRING database with a medium confidence interaction threshold (0.4). Targets were then scored based on these predicted interactions and ranked in ascending order, retaining the targets that exhibited a combined score of $\geq 0.90$ for further analysis (Supplementary Table 5). The degree values, centrality, and other parameters associated with these targets were then calculated using the Cytoscape Network Analyzer tool
(Supplementary Table 6; Figures 9, 10). The data revealed precise, relevant interactions between these targets and highlighted the PI3K/AKT/NF- $\kappa \mathrm{B}$ pathway as an essential target worthy of further investigation in the context of NAFLD treatment using ZXD. When all 122 overlapping targets were plotted, the resultant data also suggested that mitogen-activated protein kinases (MAPKs), fatty acid-binding proteins (FABPs), and matrix metalloproteinases (MMPs) may warrant future investigation in a therapeutic context.

\section{Experimental Validation of Network Pharmacology}

Significant differences $(p<0.01)$ in body weights were observed between the model and blank group rats that were fed HFD and NCD for 12 weeks, respectively (Figure 11). Furthermore, the HE-stained liver tissues of the model group rats revealed significant differences compared with the tissues of the blank group rats (Figure 12 A-B; Figures 13A,B). Specifically, liver tissue sections from the blank group rats had intact, clear boundaries; The hepatocytes had a cord-like arrangement and were radially distributed around the central vein, and the cell nuclei was clearly visible. In contrast, swelling of hepatocyte, deposition of big lipid drops and hepatic ballooning degeneration were observed in liver tissue sections from the model group rats. 

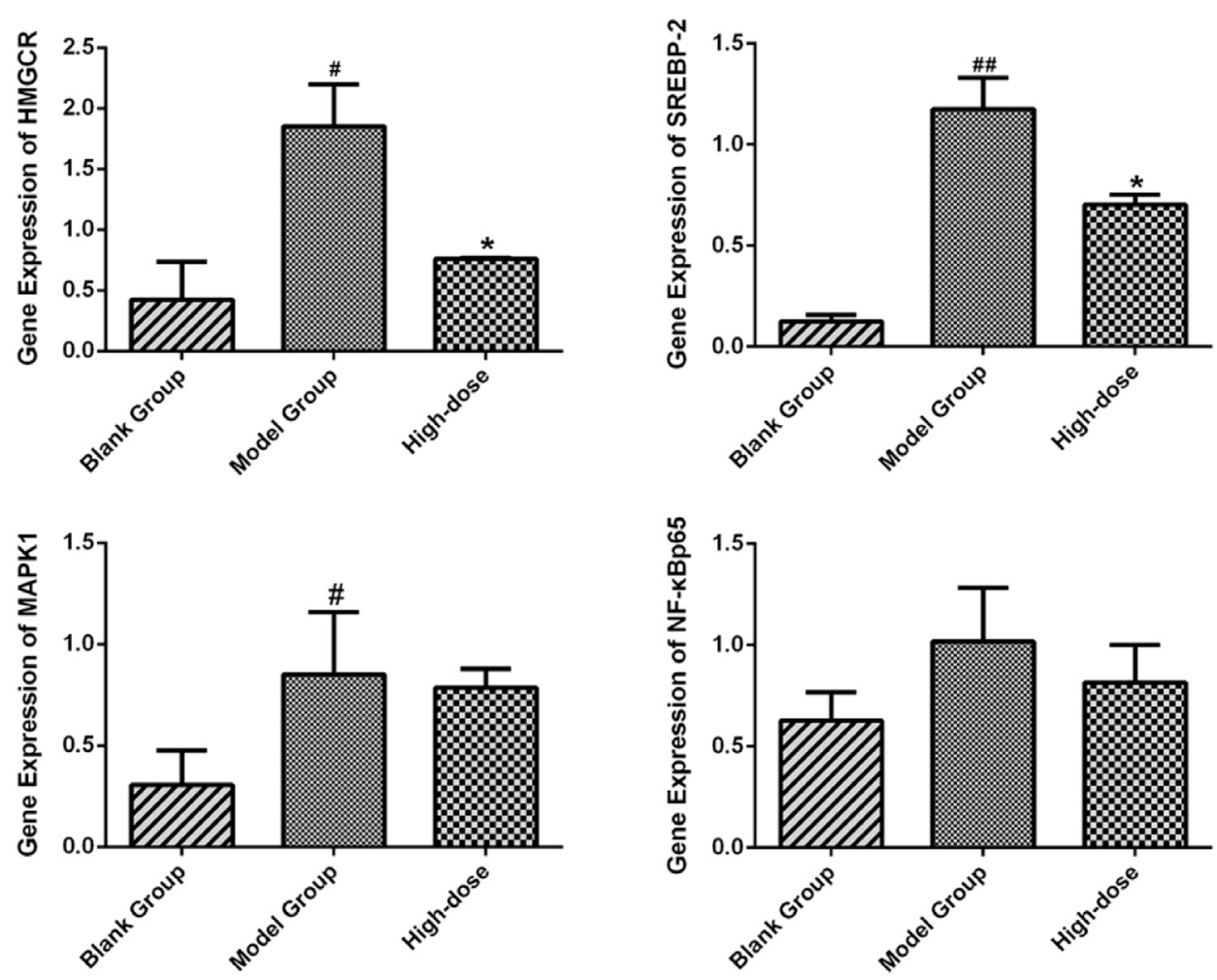

FIGURE 15 | Expression of SREBP-2, HMGCR, NF- $\kappa$ B and MAPK1 in SD rats. ${ }^{*} p<0.05$ and ${ }^{\star \star} p<0.01$ vs. model group rats; \#p $<0.05$ and \#\#p $<0.01$ vs. blank group rats.

The differences implied that the NAFLD modeling was successful. Afterwards, we treated NAFLD rats with the ethanol extract of ZXD across a range of concentrations (100, 200 and $400 \mathrm{mg} / \mathrm{kg} / \mathrm{d}$, respectively) for 4 weeks, and $\mathrm{PH}$ $(10 \mathrm{mg} / \mathrm{kg} / \mathrm{d})$ was used as a positive control. We observed significant differences in the histopathological evaluation (Figure 12 C-F; Figures 13C-F) and serum biochemical assay (Figure 14) data of different groups. The curative effect of ZXD on NAFLD was found to be dose-dependent in rats at concentrations 100, 200 and $400 \mathrm{mg} / \mathrm{kg} / \mathrm{d}$.

The liver tissues of blank, model and high-dose group rats were further conducted RT-qPCR. The results indicated that the gene expression of SREBP-2 and HMGCR of high-dose group rats decreased significantly $(p<0.05)$ in comparison with the blank group. The expression of MAPK1 and NF- $\mathrm{Bp} 65$ demonstrated an attenuation trend, which was however not significant (Figure 15). Figures 16, 17 show the amplification and melting curves of these four genes (Figures 16, 17).

\section{DISCUSSION}

\section{Identification of NAFLD-Related Targets of ZXD Treatment}

ZXD is one of the TCMFs that exhibits anti-inflammatory and lipid-lowering properties. ZXD has been used to treat NAFLD because of hyperlipidemia- and inflammation-related etiology of this disease. However, the multi-component multi-target nature of ZXD and other TCMFs has made it difficult to firmly establish the molecular mechanisms underlying their pharmacological activity. In our previous study, we used LC-MS to conduct ZXD quality control analysis (Chang et al., 2021). This analysis, together with a review of relevant pharmacologically active compounds documented in the literature, revealed that alisol A (Ho et al., 2019), alisol A 24-acetate (Li et al., 2016), alisol A 23-acetate (Lin, 2012), 25-anhydroalisol F (Bi et al., 2017), alisol F (Bi et al., 2017), and alisol M 23-acetate (Xu et al., 2018) in ZX and atractylenolide I (Chao et al., 2016; Tang et al., 2017), atractylenolide II (Chao et al., 2016), and atractylenolide III (Song et al., 2017) in BZ are highly potent despite not meeting the predictive thresholds of the TCMSP platform $(\mathrm{OB} \geq 30 \%$ and $\mathrm{DL} \geq 0.18$ ). As such, $\mathrm{OB}$ and $\mathrm{DL}$ alone are not sufficient to predict the active compounds within TCMFs.

The Swiss Target Platform was then used to identify putative NAFLD-related target genes. Further, we constructed a herbcompound-target network that identified HMGCR, MAPK1, and SREBP-2 as targets with high degree values that may be relevant in the context of ZXD-mediated NAFLD treatment.

\section{Targets Selected for Validation}

HMGCR is the rate-limiting enzyme that regulates cholesterol synthesis. Patients with NAFLD are reported to have high serum 


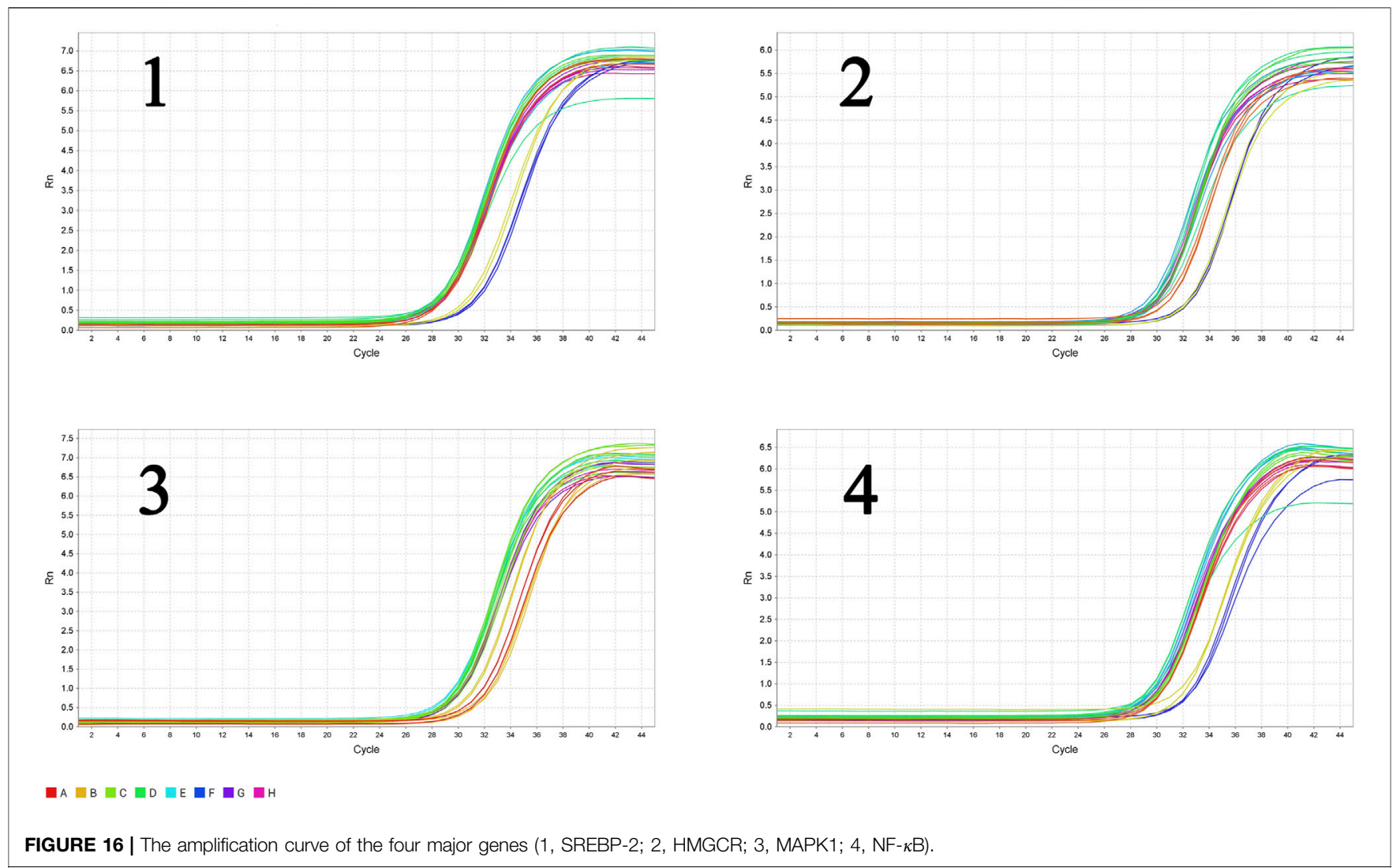

levels of total cholesterol (TC) (Puri et al., 2007; Caballero et al., 2009). The gene expression of HMGCR, however, showed an abnormally elevated serum level of TC. The expression of HMGCR is regulated by SREBP, especially by SREBP-2, a transcription factor primarily involved in the synthesis of cholesterol and fatty acid (Brown and GOLDSTEIN, 1997; Wu et al., 2013). Therefore, we hypothesized that HMGCR and SREBP-2 induce the onset and development of NAFLD by affecting the serum level of TC. There are reports that ZXD suppresses the expression of HMGCR and SREBP-2 (Zheng and WU, 2010; Dan et al., 2011). Further, the major pharmacological components of ZXD - alisol B 23-acetate and alisol A 24-acetate could bind to HMGCR competitively (Xu et al., 2016b).

MAPK signaling plays a central role in many physiological and pathological processes associated with inflammation and NAFLD (Cao et al., 2017; Jiang et al., 2019). MAPK1, MAPK8, and MAPK9 were high-scoring targets in our analysis and may thus be associated with NAFLD treatment. The pharmacologically active components of $\mathrm{BZ}$ are reported to modulate MAPK signaling (Huang et al., 2014). For instance, atractylenolide I inhibits MAPK by suppressing TLR4/MyD88 pathway activity, while atractylenolide II and III suppress inflammation by affecting MAPK phosphorylation.

$\mathrm{PI} 3 \mathrm{~K} / \mathrm{AKT} / \mathrm{NF}-\kappa \mathrm{B}$ pathways were found to be closely associated with ZXD-mediated NAFLD treatment in PPI network analyses. Since many studies (Wang et al., 2014; Fan et al., 2017) have confirmed the relationship between NAFLD and the PI3K/AKT/NF- $\kappa \mathrm{B}$ pathway, NF- $\kappa \mathrm{Bp} 65$, the critical target of the $\mathrm{PI} 3 \mathrm{~K} / \mathrm{AKT} / \mathrm{NF}-\kappa \mathrm{B}$ signaling pathway, was selected to validate our findings. Furthermore, HMGCR, MAPK1, SREBP-2, and NF- $\kappa$ Bp65 were selected for further validation through $\mathrm{RT}-\mathrm{qPCR}$.

\section{RT-qPCR Experimental Validation Using in vivo NAFLD Model in SD Rats}

The NAFLD model in rats was established using HFD and the success of modeling was verified by comparing changes in body weight and liver tissue section. The curative effect of ZXD was evaluated by histopathological evaluation and serum biochemical assays, and ZXD ameliorated lipid accumulation and treated NAFLD in a dose-dependent manner.

For validation purposes, RT-qPCR was performed to test the expression of HMGCR, MAPK1, SREBP-2, and NF- $\kappa$ Bp65. ZXD significantly attenuated the expression of HMGCR and SREBP-2 $(p<0.05)$. The expression of MAPK1 and NF- $\mathrm{kBp} 65$ demonstrated an attenuation trend, which was however not significant $(p>0.05)$. The results indicated that our network pharmacology-based prediction model of molecular mechanism is reliable to some extent.

In future studies, we plan to perform a more in-depth investigation of the molecular mechanisms of ZXD based on the gut-liver axis to study the relationship between gut 

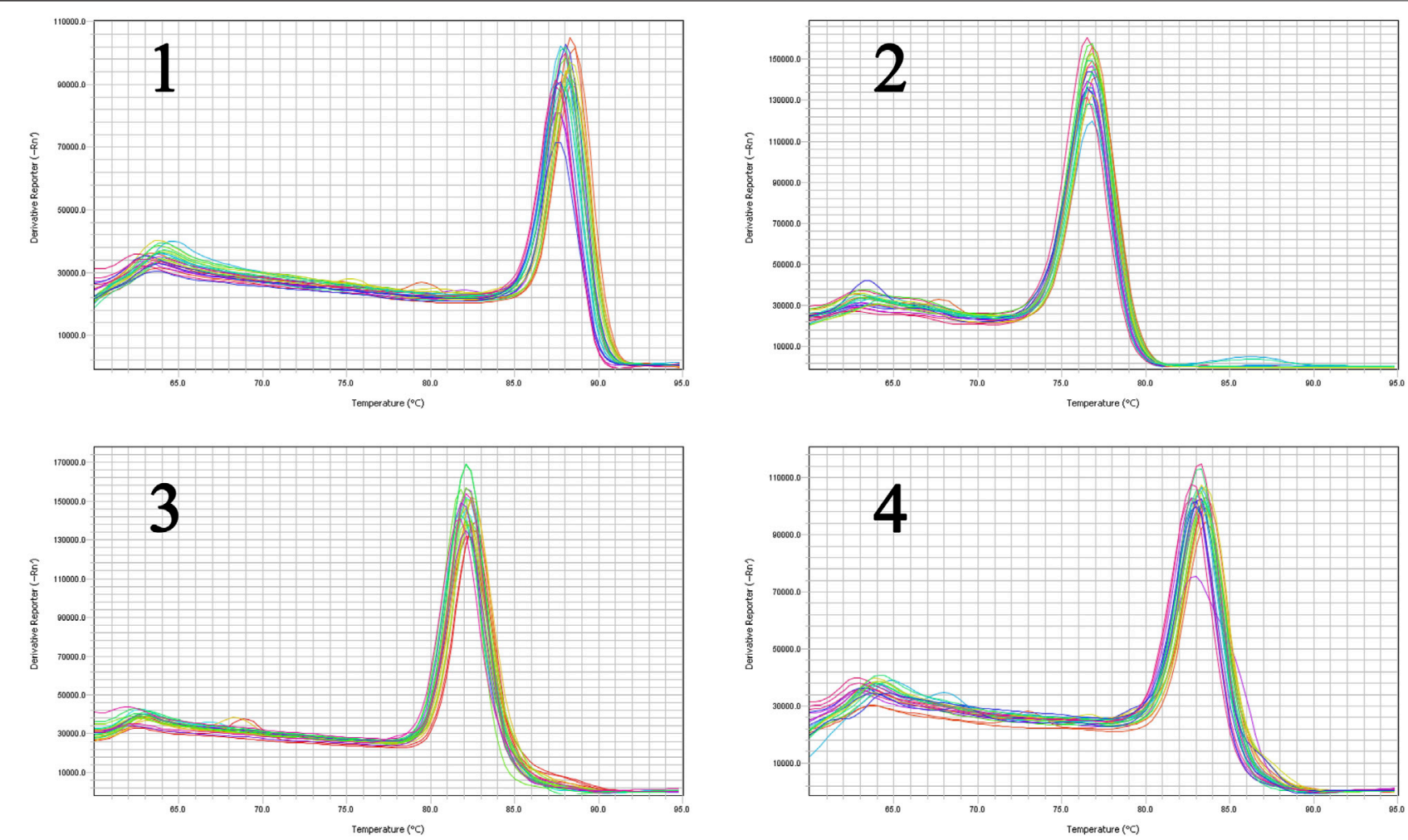

FIGURE 17 | The melting curve of the four major genes (1, SREBP-2; 2, HMGCR; 3, MAPK1; 4, NF- $\kappa$ B).

microbiota and NAFLD, using many novel databases of gut microbiota.

\section{CONCLUSION}

In conclusion, we constructed a herb-compound-target-pathway network. Using biological enrichment analyses, we determined that ZXD can treat NAFLD in part by modulating the key targets - HMGCR, SREBP-2, MAPK1, and NF- $\kappa$ Bp65. The experimental validation was performed through NAFLD model in SD rats. RTqPCR analyses revealed that ZXD could significantly attenuated the expression of the four genes. The validation results indicated that our network pharmacology-based prediction model of molecular mechanism is reliable, which can be utilized for pilot mechanism investigation of TCMFs.

\section{DATA AVAILABILITY STATEMENT}

The raw data supporting the conclusions of this article will be made available by the authors, without undue reservation, to any qualified researcher.

\section{AUTHOR CONTRIBUTIONS}

YS provided the way of thinking. JW and FZ completed the design of framework of this manuscript. JW conducted the network pharmacology study. JW, FZ and HR completed the animal experiments. XC, JW and ZL investigated the background of the present review. XC, YS and WJ were responsible for supervision, review and editing. JW wrote the manuscript and $\mathrm{FZ}$ was responsible for visualization.

\section{FUNDING}

This work was financially supported by the CAMS Innovation Fund for Medical Sciences (CIFMS) (grant No. 2016-I2M1-012).

\section{SUPPLEMENTARY MATERIAL}

The Supplementary Material for this article can be found online at: https://www.frontiersin.org/articles/10.3389/fphar.2021.722016/ full\#supplementary-material 


\section{REFERENCES}

Alex, B. (2019). UniProt: a Worldwide Hub of Protein Knowledge. Nucleic Acids Res. 47 (D1), D506-D515. doi:10.1093/nar/gky1049

Angulo, P., Kleiner, D. E., Dam-Larsen, S., Adams, L. A., Bjornsson, E. S., Charatcharoenwitthaya, P., et al. (2015). Liver Fibrosis, but No Other Histologic Features, Is Associated with Long-Term Outcomes of Patients with Nonalcoholic Fatty Liver Disease[J]. Gastroenterology 149 (2), 278-281. doi:10.1053/j.gastro.2015.04.043

Bi, X., Wang, P., Ma, Q., Han, L., Wang, X., Mu, Y., et al. (2017). AntiInflammatory Activities and Liver Protection of Alisol F and 25Anhydroalisol $\mathrm{F}$ through the Inhibition of MAPK, STAT3, and NF- $\mathrm{BB}$ Activation In Vitro and In Vivo. Molecules 22 (6), 951. doi:10.3390/ molecules22060951

Brown, M. S., and Goldstein, J. L. (1997). The SREBP Pathway: Regulation of Cholesterol Metabolism by Proteolysis of a Membrane-Bound Transcription Factor. Cell 89 (3), 331-340. doi:10.1016/s0092-8674(00)80213-5

Caballero, F., Fernández, A., De Lacy, A. M., Fernández-Checa, J. C., Caballería, J., and García-Ruiz, C. (2009). Enhanced Free Cholesterol, SREBP-2 and StAR Expression in Human NASH. J. Hepatol. 50 (4), 789-796. doi:10.1016/ j.jhep.2008.12.016

Cao, Q., Guo, J., Qian, D., Ma, H., Peng, Y., Shang, E., et al. (2017). Liposome Encapsulation Attenuated Venenum Bufonis Induced Vascular Irritation in Rabbit Ear Vein via Regulating TLR/MAPK/NF- $\mathrm{B}$ Pathway. RSC Adv. 7 (44), 27431-27440. doi:10.1039/c7ra01820g

Chang, X. Y., Li, Z. Z., Wu, J. S., Jin, W. Y., Shi, Y., et al. (2021). Identification of the Triterpenoids in the Extracts of ZeXie Decoction and Investigation of Their Effects on Nonalcoholic Fatty Liver[J]. Prog. Mod. Biomed. 21 (1), 1-12.

Chao, C. L., Huang, H. C., Lin, H. C., Chang, T. C., and Chang, W. L. (2016). Sesquiterpenes from Baizhu Stimulate Glucose Uptake by Activating AMPK and PI3K. Am. J. Chin. Med. 44 (5), 963-979. doi:10.1142/S0192415X16500531

Dan, H., Wu, J., Peng, M., Hu, X., Song, C., Zhou, Z., et al. (2011). Hypolipidemic Effects of Alismatis Rhizome on Lipid Profile in Mice Fed High-Fat Diet. Saudi Med. J. 32 (7), 701-707.

David, G., Olivier, M., and Vincent, Z. (2013). Shaping the Interaction Landscape of Bioactive Molecules[J]. Bioinformatics 29 (23), 3073-3079.

Fan, H., Ma, X., Lin, P., Kang, Q., Zhao, Z., Wang, L., et al. (2017). Scutellarin Prevents Nonalcoholic Fatty Liver Disease (NAFLD) and Hyperlipidemia via PI3K/AKT-dependent Activation of Nuclear Factor (Erythroid-Derived 2)-Like 2 (Nrf2) in Rats. Med. Sci. Monit. 23, 5599-5612. doi:10.12659/msm.907530

Gong, J., Ding, Y., Gan, Z. Y., Wang, H. W., Zhu, S. M., Li, H. Q., et al. (2018). Therapeutic Effects of Alisma Orientale Extract on Nonalcoholic Fatty Liver Disease in Rats and its Mechanism[J]. Chin. J. Comp. Med. 7 (28), 68-76.

Hamosh, A., Scott, A. F., Amberger, J., Bocchini, C., Valle, D., and Mukusick, V. A. (2002). Online Mendelian Inheritance in Man (OMIM), a Knowledgebase of Human Genes and Genetic Disorders[J]. Nucleic Acids Res. 30 (1), 52-55. doi:10.1093/nar/30.1.52

Ho, C., Gao, Y., Zheng, D., Liu, Y., Shan, S., Fang, B., et al. (2019). Alisol A Attenuates High-Fat-Diet-Induced Obesity and Metabolic Disorders via the AMPK/ACC/SREBP-1c Pathway. J. Cell Mol Med 23 (9945), 5108-5118. doi: $10.1111 /$ jcmm. 14380

Hoang, L. S., Tran, M. H., Lee, J. S., Ngo, Q. M., Woo, M. H., and Min, B. S. (2016). Inflammatory Inhibitory Activity of Sesquiterpenoids from Atractylodes Macrocephala Rhizomes. Chem. Pharm. Bull. (Tokyo) 64 (5), 507-511. doi:10.1248/cpb.c15-00805

Hong, X., Tang, H., Wu, L., and Li, L. (2010). Protective Effects of the Alisma Orientalis Extract on the Experimental Nonalcoholic Fatty Liver Disease. J. Pharm. Pharmacol. 58 (10), 1391-1398. doi:10.1211/jpp.57.10.0013

Huang, J., Guo, W., Cheung, F., Tan, H. Y., Wang, N., and Feng, Y. (2020). Integrating Network Pharmacology and Experimental Models to Investigate the Efficacy of Coptidis and Scutellaria Containing Huanglian Jiedu Decoction on Hepatocellular Carcinoma. Am. J. Chin. Med. 48 (1), 161-182. doi:10.1142/ S0192415X20500093

Huang, J. M., Zhang, G. N., Shi, Y., Zha, X., Zhu, Y., Wang, M. M., et al. (2014). Atractylenolide-I Sensitizes Human Ovarian Cancer Cells to Paclitaxel by Blocking Activation of TLR4/MyD88-dependent Pathway. Sci. Rep. 4 (3840), 3840-3847. doi:10.1038/srep03840
Jiang, J., Yan, L., Shi, Z., Wang, L., Shan, L., and Efferth, T. (2019). Hepatoprotective and Anti-inflammatory Effects of Total Flavonoids of Qu Zhi Ke (Peel of Citrus Changshan-Huyou) on Non-alcoholic Fatty Liver Disease in Rats via Modulation of NF- $\mathrm{kB}$ and MAPKs. Phytomedicine 64, 153082. doi:10.1016/j.phymed.2019.153082

Lee, A. Y., Park, W., Kang, T. W., Cha, M. H., and Chun, J. M. (2018). Network Pharmacology-Based Prediction of Active Compounds and Molecular Targets in Yijin-Tang Acting on Hyperlipidaemia and Atherosclerosis. J. Ethnopharmacol 221, 151-159. doi:10.1016/j.jep.2018.04.027

Leeson, P. D., and Springthorpe, B. (2007). The Influence of Drug-like Concepts on Decision-Making in Medicinal Chemistry. Nat. Rev. Drug Discov. 6 (11), 881-890. doi:10.1038/nrd2445

Li, X. Y., Wang, X. H., Huang, X. Q., Wu, T. T., Xu, W., and Wu, S. S. (2016). Development of a Cell-Based Peroxisome Proliferator-Activated Receptors (PPARs) Screening Model and its Application for Evaluation of Triterpenoids Isolate from Alismatis Rhizoma. Zhongguo Zhong Yao Za Zhi 41 (21), 4015-4022. doi:10.4268/cjcmm20162121

Lin, H. R. (2012). Triterpenes from Alisma Orientalis Act as Farnesoid X Receptor Agonists. Bioorg. Med. Chem. Lett. 22 (14), 4787-4792. doi:10.1016/ j.bmcl.2012.05.057

Liu, S. S., Sheng, W. L., Li, Y., Zhang, S. S., Zhu, J. J., Gao, H. M., et al. (2019). Chemical Constituents from Alismatis Rhizoma and Their Anti-inflammatory Activities In Vitro and In Vivo. Bioorg. Chem. 92, 103226. doi:10.1016/ j.bioorg.2019.103226

Puri, P., Baillie, R. A., Wiest, M. M., Mirshahi, F., Choudhury, J., Cheung, O., et al. (2007). A Lipidomic Analysis of Nonalcoholic Fatty Liver Disease. Hepatology 46 (4), 1081-1090. doi:10.1002/hep.21763

Ru, J., Li, P., Wang, J., Zhou, W., Li, B., Huang, C., et al. (2014). TCMSP: a Database of Systems Pharmacology for Drug Discovery from Herbal Medicines. J. Cheminform 6 (1), 13. doi:10.1186/1758-2946-6-13

Shannon, P., Markiel, A., Ozier, O., Baliga, N. S., Wang, J. T., Ramage, D., et al. (2003). Cytoscape: a Software Environment for Integrated Models of Biomolecular Interaction Networks. Genome Res. 13 (11), 2498-2504. doi:10.1101/gr.1239303

Song, C., Huang, X., Lu, K., Peng, M., Yu, S., and Fang, N. (2014). The Rationality of the Hypolipidemic Effect of Alismatis Rhizoma Decoction, a Classical Chinese Medicine Formula in High-Fat Diet-Induced Hyperlipidemic Mice. Iran J. Pharm. Res. 13 (2), 641-649.

Song, M. Y., Jung, H. W., Kang, S. Y., and Park, Y. K. (2017). Atractylenolide III Enhances Energy Metabolism by Increasing the SIRT-1 and PGC1 a Expression with AMPK Phosphorylation in C2C12 Mouse Skeletal Muscle Cells. Biol. Pharm. Bull. 40 (3), 339-344. doi:10.1248/bpb.b16-00853

Stelzer, G., Rosen, N., Plaschkes, I., Zimmerman, S., Twik, M., Fishilevich, S., et al. (2016). The GeneCards Suite: From Gene Data Mining to Disease Genome Sequence Analyses. Curr. Protoc. Bioinformatics 54 (1), 1-33. doi:10.1002/cpbi.5

Szklarczyk, D., Morris, J. H., Cook, H., Kuhn, M., Wyder, S., Simonovic, M., et al. (2017). The STRING Database in 2017: Quality-Controlled Protein-Protein Association Networks, Made Broadly Accessible. Nucleic Acids Res. 45 (Database issue), D362-D368. doi:10.1093/nar/gkw937

Tandon, R. K. (2013). Emergence of Non-alcoholic Fatty Liver Disease (NAFLD). J. Assoc. Physicians India 61 (7), 445-446.

Tang, X. M., Liao, Z. K., Huang, Y. W., Lin, X., and Wu, L. C. (2017). Atractylenolide I Protects against Lipopolysaccharide-Induced Disseminated Intravascular Coagulation by Anti-inflammatory and Anticoagulation Effect. Asian Pac. J. Trop. Med. 10 (6), 582-587. doi:10.1016/j.apjtm.2017.06.007

Than, N. N., and Newsome, P. N. (2015). A Concise Review of Non-alcoholic Fatty Liver Disease[J]. Atherosclerosis 239 (1), 192-202. doi:10.1016/ j.atherosclerosis.2015.01.001

Wang, C., Chi, Y., Li, J., Miao, Y., Li, S., Su, W., et al. (2014). FAM3A Activates PI3K p110a/Akt Signaling to Ameliorate Hepatic Gluconeogenesis and Lipogenesis. Hepatology 59 (5), 1779-1790. doi:10.1002/hep.26945

Wu, C., Jing, M., Yang, L., Jin, L., Ding, Y., Lu, J., et al. (2018). Alisol A 24-acetate Ameliorates Nonalcoholic Steatohepatitis by Inhibiting Oxidative Stress and Stimulating Autophagy through the AMPK/mTOR Pathway. Chem. Biol. Interact 291, 111-119. doi:10.1016/j.cbi.2018.06.005

Wu, J. S., Chang, X. Y., Li, Z. Z., Jin, W. Y., Shi, Y., et al. (2021). Research Progress on Molecular Mechanism of Lipid-Lowering and Anti-inflammatory Effect of Zexietang[J]. Chin. J. Exp. Traditional Med. Formulae 27 (2), 224-232. 
Wu, N., Sarna, L. K., Hwang, S. Y., Zhu, Q., Wang, P., Siow, Y. L., et al. (2013). Activation of 3-Hydroxy-3-Methylglutaryl Coenzyme A (HMG-CoA) Reductase during High Fat Diet Feeding. Biochim. Biophys. Acta 1832 (10), 1560-1568. doi:10.1016/j.bbadis.2013.04.024

Xu, F., Lu, C., Wu, Q., Gu, W., Chen, J., Fang, F., et al. (2018). Studies on the LipidRegulating Mechanism of Alisol-Based Compounds on Lipoprotein Lipase. Bioorg. Chem. 80 (2018), 347-360. doi:10.1016/j.bioorg.2018.07.001

Xu, F., Yu, H., Lu, C., Chen, J., and Gu, W. (2016). The Cholesterol-Lowering Effect of Alisol Acetates Based on HMG-CoA Reductase and its Molecular Mechanism[J]. Evid.-based Complement. Altern. Med. 2016 (6), 1-11.

Xu, F., Yu, H., Lu, C., Chen, J., and Gu, W. (2016). The Cholesterol-Lowering Effect of Alisol Acetates Based on HMG-CoA Reductase and its Molecular Mechanism[J]. Evidence-Based Complement. Altern. Med. 2016, 1-11.

Xu, X., Zhang, W., Huang, C., Li, Y., Yu, H., Wang, Y., et al. (2012). A Novel Chemometric Method for the Prediction of Human Oral Bioavailability. Int. J. Mol. Sci. 13 (6), 6964-6982. doi:10.3390/ijms13066964

Yang, J. M., Sun, Y., Wang, M., Zhang, X. L., Zhang, S. J., Gao, Y. S., et al. (2019). Regulatory Effect of a Chinese Herbal Medicine Formula on Non-alcoholic Fatty Liver Disease. World J. Gastroenterol. 25 (34), 5105-5119. doi:10.3748/ wjg.v25.i34.5105

Zhang, R., Zhu, X., Bai, H., and Ning, K. (2019). Network Pharmacology Databases for Traditional Chinese Medicine: Review and Assessment. Front. Pharmacol. 10, 123. doi:10.3389/fphar.2019.00123

Zheng, C., and Wu, S. (2010). Screening Lipid-Reducing Active Components from Rhizoma Alismatis by Computer Simulation[J]. Rehabil. Med. 20 (3), 34-36.
Zhou, Y., Zhou, B., Pache, L., Chang, M., Khodabakhshi, A. H., Tanaseichuk, O., et al. (2019). Metascape Provides a Biologist-Oriented Resource for the Analysis of SystemsLevel Datasets. Nat. Commun. 10 (1), 1523. doi:10.1038/s41467-019-09234-6

Zuo, H., Zhang, Q., Su, S., Chen, Q., Yang, F., and Hu, Y. (2018). A Network Pharmacology-Based Approach to Analyse Potential Targets of Traditional Herbal Formulas: An Example of Yu Ping Feng Decoction[J]. Sci. Rep. 8 (1), 11418. doi:10.1038/s41598-018-29764-1

Conflict of Interest: The authors declare that the research was conducted in the absence of any commercial or financial relationships that could be construed as a potential conflict of interest.

Publisher's Note: All claims expressed in this article are solely those of the authors and do not necessarily represent those of their affiliated organizations, or those of the publisher, the editors and the reviewers. Any product that may be evaluated in this article, or claim that may be made by its manufacturer, is not guaranteed or endorsed by the publisher.

Copyright (C) $2021 \mathrm{Wu}$, Zhang, Ruan, Chang, Wang, Li, Jin and Shi. This is an openaccess article distributed under the terms of the Creative Commons Attribution License (CC BY). The use, distribution or reproduction in other forums is permitted, provided the original author(s) and the copyright owner(s) are credited and that the original publication in this journal is cited, in accordance with accepted academic practice. No use, distribution or reproduction is permitted which does not comply with these terms. 